TRANSACTIONS OF THE

AMERICAN MATHEMATICAL SOCIETY

Volume 362, Number 10, October 2010, Pages 5483-5500

S 0002-9947(2010)05022-6

Article electronically published on May 19, 2010

\title{
EXTENSIONS OF LINKING SYSTEMS AND FUSION SYSTEMS
}

\author{
BOB OLIVER
}

\begin{abstract}
We correct two errors in the statement and proof of a theorem in an earlier paper (2007), and at the same time extend that result to a more general theorem about extensions of $p$-local finite groups. Other special cases of this theorem have already been shown in two later papers, so we feel it will be useful to have this more general result in the literature.
\end{abstract}

This paper has two purposes: to correct some errors in the statement and proof of a theorem in the earlier paper [5], and also to prove a more general version of this theorem, describing (very roughly) how to construct extensions of fusion and linking systems by groups of outer automorphisms. Special cases of this construction have been used in at least two papers written since [5].

When $G$ is a finite group and $S \in \operatorname{Syl}_{p}(G)$, the fusion category of $G$ is the category $\mathcal{F}_{S}(G)$ whose objects consist of all subgroups of $S$, and where

$$
\operatorname{Mor}_{\mathcal{F}_{S}(G)}(P, Q)=\operatorname{Hom}_{G}(P, Q) \stackrel{\text { def }}{=}\left\{c_{g} \in \operatorname{Hom}(P, Q) \mid g \in G, g P g^{-1} \leq Q\right\} .
$$

This gives a means of encoding the $p$-local structure of $G$ : the conjugacy relations among the $p$-subgroups of $G$. The centric linking category of $G$ is a closely related category which (among other things) provides a link between the fusion in $G$ and the homotopy type of its $p$-completed classifying space. These categories motivated the definition by Puig [10] of abstract fusion systems, and by Broto, Levi, and Oliver [3] of abstract linking systems.

The main theorem in this paper (Theorem 9) describes how to construct certain types of extensions of abstract fusion and linking systems. The special case shown in [5. Theorem 4.6] shows how to extend a linking system by a $p$-group of outer automorphisms. Other special cases were used by Castellana and Libman [6] to construct wreath products of linking systems, and by Andersen, Oliver, and Ventura [1] to construct exotic fusion and linking systems under certain hypotheses. Since all three of these constructions have very similar proofs, it should be useful to have one reference which covers all of these cases, and hopefully any others which might be needed in the future.

There was an omission in the statement of [5, Theorem 4.6], in that the group $S$ must be assumed to act on $\mathcal{L}_{0}$ via isotypical automorphisms (Definition 5 ). Without this assumption, it need not induce an action on the fusion system $\mathcal{F}_{0}$. The error in the proof of the theorem occurs in Step 4. In that step, a certain property of

Received by the editors November 21, 2008 and, in revised form, February 13, 2009.

2000 Mathematics Subject Classification. Primary 55R35; Secondary 20D20, 20 E22.

Key words and phrases. Classifying spaces, Sylow subgroups, fusion, extensions.

The author was partially supported by UMR 7539 of the CNRS.

(C)2010 American Mathematical Society Reverts to public domain 28 years from publication 
subgroups not in a family $\mathcal{H}$ was proven using a result shown in Step 3 - a result shown there only for subgroups which are in the family $\mathcal{H}$.

In most cases, our main interest is to construct extensions of fusion systems. However, when trying to do this, one quickly discovers that a fusion system alone does not contain enough information to construct extensions, at least not in a straightforward way. This is why the results in [5], 6], and [1] are all stated in terms of linking systems. Furthermore, the extensions $\mathcal{L}_{0} \unlhd \mathcal{L}$ of linking systems which we construct are such that the geometric realization $\left|\mathcal{L}_{0}\right|$ has the homotopy type of a finite covering space of $|\mathcal{L}|$ - with covering group the group of outer automorphisms by which we extended. This is not in general the case for extensions of the fusion systems.

We now recall the definitions of abstract fusion and linking systems, and their basic properties which will be needed later.

An abstract fusion system over a finite $p$-group $S$ is a category $\mathcal{F}$ such that $\mathrm{Ob}(\mathcal{F})$ is the set of all subgroups of $S$, and such that for all $P, Q \leq S$,

- $\operatorname{Hom}_{S}(P, Q) \subseteq \operatorname{Hom}_{\mathcal{F}}(P, Q) \subseteq \operatorname{Inj}(P, Q)$;

- each $\varphi \in \operatorname{Hom}_{\mathcal{F}}(P, Q)$ is the composite of an $\mathcal{F}$-isomorphism followed by an inclusion.

Some additional conditions are needed to make this very useful.

Definition 1 ([10], 3$])$. Let $\mathcal{F}$ be a fusion system over a $p$-group $S$.

- Two subgroups $P, Q \leq S$ are $\mathcal{F}$-conjugate if they are isomorphic as objects of the category $\mathcal{F}$.

- A subgroup $P \leq S$ is fully centralized in $\mathcal{F}$ if $\left|C_{S}(P)\right| \geq\left|C_{S}\left(P^{\prime}\right)\right|$ for all $P^{\prime} \leq S$ which is $\mathcal{F}$-conjugate to $P$.

- A subgroup $P \leq S$ is fully normalized in $\mathcal{F}$ if $\left|N_{S}(P)\right| \geq\left|N_{S}\left(P^{\prime}\right)\right|$ for all $P^{\prime} \leq S$ which is $\mathcal{F}$-conjugate to $P$.

- $\mathcal{F}$ is saturated if the following two conditions hold:

(I) For each $P \leq S$ which is fully normalized in $\mathcal{F}, P$ is fully centralized in $\mathcal{F}$ and $\operatorname{Aut}_{S}(P) \in \operatorname{Syl}_{p}\left(\operatorname{Aut}_{\mathcal{F}}(P)\right)$.

(II) For each $P \leq S$ and $\varphi \in \operatorname{Hom}_{\mathcal{F}}(P, S)$ such that $\varphi P$ is fully centralized, if we set

$$
N_{\varphi}=\left\{g \in N_{S}(P) \mid \varphi c_{g} \varphi^{-1} \in \operatorname{Aut}_{S}(\varphi P)\right\},
$$

then there is a $\bar{\varphi} \in \operatorname{Hom}_{\mathcal{F}}\left(N_{\varphi}, S\right)$ such that $\left.\bar{\varphi}\right|_{P}=\varphi$.

More generally, when $\mathcal{H}$ is a set of subgroups of $S$ closed under $\mathcal{F}$-conjugacy, $\mathcal{F}$ is $\mathcal{H}$-saturated if axioms (I) and (II) hold for all $P \in \mathcal{H}$.

The main objects of interest here are the saturated fusion systems. For example, $\mathcal{F}_{S}(G)$ is saturated for any finite group $G$ and any $S \in \operatorname{Syl}_{p}(G)$. Axioms (I) and (II) follow mostly as consequences of the Sylow theorems (cf. [3, Proposition 1.3]).

We will need to refer frequently to the following classes of subgroups in a fusion system.

Definition 2. Let $\mathcal{F}$ be a fusion system over a $p$-group $S$.

- A subgroup $P \leq S$ is $\mathcal{F}$-centric if it is fully centralized and $C_{S}(P)=Z(P)$;

- $P \leq S$ is $\mathcal{F}$-radical if $O_{p}\left(\operatorname{Aut}_{\mathcal{F}}(P)\right)=\operatorname{Inn}(P)$; 
- $P \leq S$ is $\mathcal{F}$-quasicentric if for each $P^{\prime} \leq S$ which is fully centralized and $\mathcal{F}$ conjugate to $P$, each $P^{\prime} \leq Q \leq P^{\prime} \cdot C_{S}\left(P^{\prime}\right)$, and each $\alpha \in \operatorname{Aut}_{\mathcal{F}}(Q)$ such that $\left.\alpha\right|_{P^{\prime}}=\mathrm{Id}, \alpha$ has $p$-power order.

We now turn to abstract linking systems. As explained above, they seem to be the most natural structures for describing extensions of the type which we are looking at in this paper. For any finite group $G$, let $\mathcal{T}(G)$ denote the transporter category of $G$ : the category with $\operatorname{Ob}(\mathcal{T}(G))$ the set of all subgroups of $G$, and where for each $P, Q \leq G$,

$$
\operatorname{Mor}_{\mathcal{T}(G)}(P, Q)=N_{G}(P, Q) \stackrel{\text { def }}{=}\left\{g \in G \mid g P g^{-1} \leq Q\right\}
$$

(the transporter set). When $\mathcal{H}$ is a set of subgroups of $G$, then $\mathcal{T}_{\mathcal{H}}(G) \subseteq \mathcal{T}(G)$ denotes the full subcategory with object set $\mathcal{H}$.

Definition 3 ([3, Definition 1.7] \& [4, Definition 3.3]). Let $\mathcal{F}$ be a fusion system over a $p$-group $S$. A linking system associated to $\mathcal{F}$ is a finite category $\mathcal{L}$, together with a pair of functors

$$
\mathcal{T}_{\mathrm{Ob}(\mathcal{L})}(S) \stackrel{\delta}{\longrightarrow} \mathcal{L} \stackrel{\pi}{\longrightarrow} \mathcal{F},
$$

satisfying the following conditions:

(A) $\operatorname{Ob}(\mathcal{L})$ is a set of subgroups of $S$ closed under $\mathcal{F}$-conjugacy and overgroups and includes all subgroups which are $\mathcal{F}$-centric and $\mathcal{F}$-radical. Each object in $\mathcal{L}$ is isomorphic (in $\mathcal{L}$ ) to one which is fully centralized in $\mathcal{F}$. Also, $\delta$ is the identity on objects, and $\pi$ is the inclusion on objects. For each $P, Q \in \operatorname{Ob}(\mathcal{L})$ such that $P$ is fully centralized in $\mathcal{F}, C_{S}(P)$ acts freely on $\operatorname{Mor}_{\mathcal{L}}(P, Q)$ via $\delta_{P, P}$ and right composition, and $\pi_{P, Q}$ induces a bijection

$$
\operatorname{Mor}_{\mathcal{L}}(P, Q) / C_{S}(P) \stackrel{\cong}{\longrightarrow} \operatorname{Hom}_{\mathcal{F}}(P, Q) \text {. }
$$

(B) For each $P, Q \in \mathrm{Ob}(\mathcal{L})$ and each $g \in N_{S}(P, Q), \pi_{P, Q}$ sends $\delta_{P, Q}(g) \in$ $\operatorname{Mor}_{\mathcal{L}}(P, Q)$ to $c_{g} \in \operatorname{Hom}_{\mathcal{F}}(P, Q)$.

(C) For all $\varphi \in \operatorname{Mor}_{\mathcal{L}}(P, Q)$ and all $g \in P$, the diagram

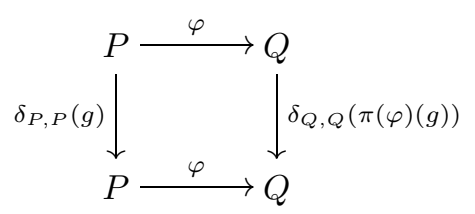

commutes in $\mathcal{L}$.

The main differences between this definition and those in 3 and 4 are that it is more flexible on the set of objects in $\mathcal{L}$, and that we define here $\delta$ as a functor on the transporter category of $S$. That $\delta$ can be defined on $\mathcal{T}_{\mathrm{Ob}(\mathcal{L})}(S)$ follows as a consequence of the earlier definitions (see [3, Proposition 1.11] and [4, Lemma 3.7]), and including it in the definition allows us to drop axiom $(\mathrm{D})_{q}$ in [4, Definition 3.3]. We will see shortly (in Proposition $4(\mathrm{~g})$ ) that all objects in a linking system $\mathcal{L}$ must be quasicentric.

The condition that $\mathcal{L}$ be closed under overgroups could perhaps be dropped. But it simplifies the proof of Proposition 4 below and is needed in any case in the hypotheses of the main theorem. Also, it is difficult to imagine a situation where we might need a linking system which is not closed under overgroups. 
The reason for assuming $\mathcal{L}$ contains all subgroups which are $\mathcal{F}$-centric and $\mathcal{F}$ radical originates with [4, Theorem 3.5], which says that if $\mathcal{L}^{\prime} \subseteq \mathcal{L}$ are two linking systems associated to the same fusion system, such that $\operatorname{Ob}\left(\mathcal{L}^{\prime}\right)$ contains all $\mathcal{F}$ centric $\mathcal{F}$-radical subgroups and $\operatorname{Ob}(\mathcal{L})$ is contained in the set of all $\mathcal{F}$-quasicentric subgroups, then the geometric realizations of these two categories are homotopy equivalent. In other words, this seems to be the minimal set of objects needed to get the information which one needs from a linking system. But as we will see shortly, this also plays an important role in the proof of Theorem 9 below.

In general, when $\mathcal{L}$ is a linking system over $S$, and $P \leq Q$ are both objects in $\mathcal{L}$, we define $\iota_{P}^{Q}=\delta_{P, Q}(1)$, where $\delta: \mathcal{T}_{\mathrm{Ob}(\mathcal{L})}(S) \longrightarrow \mathcal{L}$ is the functor in the definition of $\mathcal{L}$. We regard these morphisms as the inclusion morphisms in $\mathcal{L}$.

Proposition 4. The following hold for any linking system $\mathcal{L}$ associated to a saturated fusion system $\mathcal{F}$ over a p-group $S$.

(a) For each $P, Q \in \mathrm{Ob}(\mathcal{L})$, the subgroup $E(P) \stackrel{\text { def }}{=} \operatorname{Ker}\left[\operatorname{Aut}_{\mathcal{L}}(P) \longrightarrow \operatorname{Aut}_{\mathcal{F}}(P)\right]$ acts freely on $\operatorname{Mor}_{\mathcal{L}}(P, Q)$ via right composition, and $\pi_{P, Q}$ induces a bijection

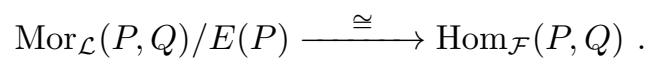

(b) For every morphism $\psi \in \operatorname{Mor}_{\mathcal{L}}(P, Q)$, and every $P_{0}, Q_{0} \in \operatorname{Ob}(\mathcal{L})$ such that $P_{0} \leq P, Q_{0} \leq Q$, and $\pi(\psi)\left(P_{0}\right) \leq Q_{0}$, there is a unique morphism $\left.\psi\right|_{P_{0}, Q_{0}} \in$ $\operatorname{Mor}_{\mathcal{L}}\left(P_{0}, Q_{0}\right)$ (the "restriction" of $\psi$ ) such that $\psi \circ \iota_{P_{0}}^{P}=\left.\iota_{Q_{0}}^{Q} \circ \psi\right|_{P_{0}, Q_{0}}$.

(c) The functor $\delta$ is injective on all morphism sets.

(d) If $P \in \operatorname{Ob}(\mathcal{L})$ is fully normalized in $\mathcal{F}$, then $\delta_{P}\left(N_{S}(P)\right) \in \operatorname{Syl}_{p}\left(\operatorname{Aut}_{\mathcal{L}}(P)\right)$.

(e) Let $P, Q, \bar{P}, \bar{Q} \in \operatorname{Ob}(\mathcal{L})$ and $\psi \in \operatorname{Mor}_{\mathcal{L}}(P, Q)$ be such that $P \unlhd \bar{P}, Q \leq \bar{Q}$, and for each $g \in \bar{P}$ there is $h \in \bar{Q}$ such that $\iota_{Q}^{\bar{Q}} \circ \psi \circ \delta_{P}(g)=\delta_{Q, \bar{Q}}(h) \circ \psi$. Then there is a unique morphism $\bar{\psi} \in \operatorname{Mor}_{\mathcal{L}}(\bar{P}, \bar{Q})$ such that $\left.\bar{\psi}\right|_{P, Q}=\psi$.

(f) All morphisms in $\mathcal{L}$ are monomorphisms and epimorphisms in the categorical sense.

(g) All objects in $\mathcal{L}$ are $\mathcal{F}$-quasicentric. In other words, if $P \in \operatorname{Ob}(\mathcal{L})$ is fully centralized in $\mathcal{F}$, and $P \leq Q \leq P C_{S}(P)$, then each automorphism $\alpha \in \operatorname{Aut}_{\mathcal{F}}(Q)$ such that $\left.\alpha\right|_{P}=\operatorname{Id}_{P}$ has p-power order.

Proof. (a) Fix $P, Q \in \mathrm{Ob}(\mathcal{L})$. By axiom $(\mathrm{A})$, there is a subgroup $P^{*} \in \mathrm{Ob}(\mathcal{L})$ fully centralized in $\mathcal{F}$ and an isomorphism $\alpha \in \operatorname{Iso}_{\mathcal{L}}\left(P^{*}, P\right)$. Set $\beta=\pi(\alpha) \in \operatorname{Iso}_{\mathcal{F}}\left(P^{*}, P\right)$, and consider the following commutative squares:

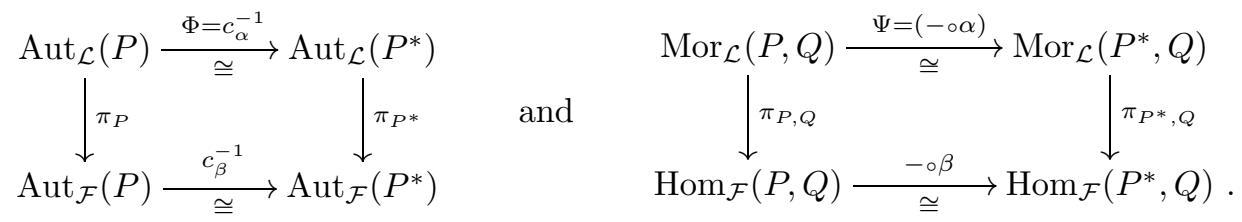

By the commutativity of the first square, $\Phi$ sends $E(P)=\operatorname{Ker}\left(\pi_{P, P}\right)$ onto $E\left(P^{*}\right)=$ $\operatorname{Ker}\left(\pi_{P^{*}, P^{*}}\right)$. By $(\mathrm{A}), E\left(P^{*}\right)$ acts freely on $\operatorname{Mor}_{\mathcal{L}}\left(P^{*}, Q\right)$, and $\pi_{P^{*}, Q}$ is the orbit map of this action. Since $\Psi(\psi \circ \chi)=\Psi(\psi) \circ \Phi(\chi)$ for $\psi \in \operatorname{Mor}_{\mathcal{L}}(P, Q)$ and $\chi \in \operatorname{Aut}_{\mathcal{L}}(P)$, it follows that $E(P)$ acts freely on $\operatorname{Mor}_{\mathcal{L}}(P, Q)$ and $\pi_{P, Q}$ is the orbit map of that action. 
(b) Fix $\psi \in \operatorname{Mor}_{\mathcal{L}}(P, Q)$, and $P_{0} \leq P, Q_{0} \leq Q$ as above, and set $\varphi=\left.\pi(\psi)\right|_{P_{0}, Q_{0}} \in$ $\operatorname{Hom}_{\mathcal{F}}\left(P_{0}, Q_{0}\right)$. Let $\psi_{1} \in \operatorname{Mor}_{\mathcal{L}}\left(P_{0}, Q_{0}\right)$ be any morphism such that $\pi\left(\psi_{1}\right)=\varphi$. Then $\pi\left(\iota_{Q_{0}}^{Q} \circ \psi_{1}\right)=\pi\left(\psi \circ \iota_{P_{0}}^{P}\right)$, so by $(\mathbf{a})$, there is $\chi \in E\left(P_{0}\right)$ such that $\iota_{Q_{0}}^{Q} \circ \psi_{1} \circ \chi=$ $\psi \circ \iota_{P_{0}}^{P}$. We can thus take $\left.\psi\right|_{P_{0}, Q_{0}}=\psi_{1} \circ \chi$.

Now assume $\psi_{0}$ and $\psi_{0}^{\prime}$ are two such restrictions; thus $\iota_{Q_{0}}^{Q} \circ \psi_{0}=\iota_{Q_{0}}^{Q} \circ \psi_{0}^{\prime}$. By (a) again, there is $\chi \in E\left(P_{0}\right)$ such that $\psi_{0}^{\prime}=\psi_{0} \circ \chi$. But then $\chi=\operatorname{Id}_{P_{0}}$ since $E\left(P_{0}\right)$ acts freely on $\operatorname{Mor}_{\mathcal{L}}\left(P_{0}, Q\right)$, and thus $\psi_{0}=\psi_{0}^{\prime}$.

(c) For any pair of objects $P$ and $Q, \pi \circ \delta_{P, Q}$ sends $N_{S}(P, Q)=\operatorname{Mor}_{\mathcal{T}(S)}(P, Q)$ onto $\operatorname{Hom}_{S}(P, Q) \cong N_{S}(P, Q) / C_{S}(P)$. By (a), $E(P)$ acts freely on $\pi^{-1}\left(\operatorname{Hom}_{S}(P, Q)\right)$. Hence $\delta_{P, Q}$ is injective if $\delta_{P}$ sends $C_{S}(P)$ injectively into $E(P)$. By (A), this is the case whenever $P$ is fully centralized in $\mathcal{F}$.

If $P$ is not fully centralized, then choose $P^{*}$ which is fully centralized, and $\varphi \in \operatorname{Iso}_{\mathcal{F}}\left(P, P^{*}\right)$. By the extension axiom (II), there is $\bar{\varphi} \in \operatorname{Hom}_{\mathcal{F}}\left(C_{S}(P) \cdot P, S\right)$ such that $\left.\bar{\varphi}\right|_{P}=\varphi$. Set $R=\operatorname{Im}(\bar{\varphi})$; thus $R \leq C_{S}\left(P^{*}\right) \cdot P^{*}$. Choose $\psi \in \operatorname{Iso}_{\mathcal{L}}\left(C_{S}(P) \cdot P, R\right)$ such that $\pi(\psi)=\bar{\varphi}$, and set $\psi_{0}=\left.\psi\right|_{P, P^{*}}$.

By axiom (C), for each $g \in C_{S}(P), \psi \circ \delta_{C_{S}(P) \cdot P}(g) \circ \psi^{-1}=\delta_{R}(\bar{\varphi}(g))$. Upon restricting to $P$ and $P^{*}$, this implies $\psi_{0} \circ \delta_{P}(g) \circ \psi_{0}^{-1}=\delta_{P^{*}}(\bar{\varphi}(g))$. If $g \neq 1$, then $\bar{\varphi}(g) \neq 1$, and hence $\delta_{P^{*}}(\bar{\varphi}(g)) \neq \mathrm{Id}$ since $P^{*}$ is fully centralized. Since $\psi_{0}$ is an isomorphism, this implies $\delta_{P}(g) \neq \mathrm{Id}$, and thus that $\delta_{P}$ is injective on $C_{S}(P)$.

(d) If $P \in \operatorname{Ob}(\mathcal{L})$ is fully normalized in $\mathcal{F}$, then $\operatorname{Aut}_{S}(P) \in \operatorname{Syl}_{p}\left(\operatorname{Aut}_{\mathcal{F}}(P)\right)$,

$$
\operatorname{Aut}_{\mathcal{F}}(P) \cong \operatorname{Aut}_{\mathcal{L}}(P) / \delta_{P}\left(C_{S}(P)\right) \quad \text { and } \quad \operatorname{Aut}_{S}(P) \cong N_{S}(P) / C_{S}(P),
$$

and hence $\delta_{P}\left(N_{S}(P)\right) \in \operatorname{Syl}_{p}\left(\operatorname{Aut}_{\mathcal{L}}(P)\right)$.

(e) Set $\varphi=\pi(\psi), Q_{0}=\varphi(P), \psi_{0}=\left.\psi\right|_{P, Q_{0}} \in \operatorname{Iso}_{\mathcal{L}}\left(P, Q_{0}\right)$, and $\bar{Q}_{0}=N_{\bar{Q}}\left(Q_{0}\right)$. If $g \in \bar{P}$ and $h \in \bar{Q}$ are such that $\iota_{Q}^{\bar{Q}} \circ \psi \circ \delta_{P}(g)=\delta_{Q, \bar{Q}}(h) \circ \psi$, then for each $x \in P$, $\varphi\left(g x g^{-1}\right)=h \varphi(x) h^{-1} \in Q_{0}$, and thus $h \in \bar{Q}_{0}$. Also,

$$
\psi_{0} \circ \delta_{P}(g)=\left.\left(\psi \circ \delta_{P}(g)\right)\right|_{P, Q_{0}}=\left.\left(\delta_{Q, Q^{*}}(h) \circ \psi\right)\right|_{P, Q_{0}}=\delta_{Q_{0}}(h) \circ \psi_{0}
$$

by the uniqueness of restrictions in (b). We are thus reduced to proving this point when $\psi$ is an isomorphism and $Q \unlhd \bar{Q}$.

Now assume $\psi \in \operatorname{Iso}_{\mathcal{L}}(P, Q)$; thus $\psi \circ \delta_{P}(\bar{P}) \circ \psi^{-1} \leq \delta_{Q}(\bar{Q})$. If $Q$ is fully centralized in $\mathcal{F}$, then axiom (II) for the saturated fusion system $\mathcal{F}$ implies that $\varphi=\pi(\psi)$ extends to a homomorphism $\bar{\varphi} \in \operatorname{Hom}_{\mathcal{F}}(\bar{P}, S)$. Choose $\widehat{\psi} \in \operatorname{Mor}_{\mathcal{L}}(\bar{P}, S)$ such that $\pi(\widehat{\psi})=\bar{\varphi}$. Then $\pi\left(\left.\widehat{\psi}\right|_{P, Q}\right)=\pi(\psi)$. Since $Q$ is fully centralized, there is $g \in$ $C_{S}(Q)$ such that $\psi=\left.\delta_{Q}(g) \circ \widehat{\psi}\right|_{P, Q}$ by axiom (A) (applied to $\delta_{Q, P}$ and the morphisms $\psi^{-1}$ and $\left.\left(\left.\widehat{\psi}\right|_{P, Q}\right)^{-1}\right)$. Set $\bar{\psi}=\delta_{S}(g) \circ \widehat{\psi}$; then $\left.\bar{\psi}\right|_{P, Q}=\psi$. Also, $\pi(\bar{\psi})(\bar{P}) \leq \bar{Q}$ by $(\mathrm{C})$ and the original assumption on $\psi$, and so $\bar{\psi}$ restricts to a morphism in $\operatorname{Mor}_{\mathcal{L}}(\bar{P}, \bar{Q})$ by (b). Note that this is an isomorphism if $\psi \circ \delta_{P}(\bar{P}) \circ \psi^{-1}=\delta_{Q}(\bar{Q})$.

Now assume $Q$ is not fully centralized. Choose $R$ which is $\mathcal{F}$-conjugate to $P$ and $Q$ and fully normalized in $\mathcal{F}$. Fix an isomorphism $\varphi \in \operatorname{Iso}_{\mathcal{L}}(Q, R)$. Then $\varphi \delta_{Q}(\bar{Q}) \varphi^{-1}$ is a $p$-subgroup of $\operatorname{Aut}_{\mathcal{L}}(R)$, so by $(\mathbf{d})$, there is $\chi \in \operatorname{Aut}_{\mathcal{L}}(R)$ such that $(\chi \varphi) \delta_{Q}(\bar{Q})(\chi \varphi)^{-1}=\delta_{R}(\bar{R})$ for some $\bar{R} \leq N_{S}(R)$. We just showed that $\chi \varphi$ and $\chi \varphi \psi$ extend to morphisms $\bar{\varphi} \in \operatorname{Iso}_{\mathcal{L}}(\bar{Q}, \bar{R})$ and $\bar{\psi} \in \operatorname{Mor}_{\mathcal{L}}(\bar{P}, \bar{R})$, and so $\bar{\varphi}^{-1} \circ \bar{\psi} \in$ $\operatorname{Mor}_{\mathcal{L}}(\bar{P}, \bar{Q})$ extends $\psi$. This proves (e), except for the uniqueness, which will follow from (f). 
(f) We claim that $\mathcal{L}$ is a transporter system in the sense of [8, Definition 3.1]. Once this has been shown, then (f) follows from [8, Lemma 3.2(b,d)].

Points (A1) and (C) in [8, Definition 3.1] hold for $\mathcal{L}$ by definition, while (II) holds by (e) (it requires only the existence of $\bar{\psi}$ and not uniqueness), and (I) follows from (d). Point (B) holds by axiom (B) here, together with the injectivity of $\delta$ shown in $(\mathbf{c})$.

Point (A2) holds by (a), except for showing that for all $P, Q \in \operatorname{Ob}(\mathcal{L}), E(Q)$ acts freely on $\operatorname{Mor}_{\mathcal{L}}(P, Q)$ by left composition. Since this property depends only on the isomorphism class of $Q$ in $\mathcal{L}$, it suffices to prove it when $Q$ is fully centralized, and hence when $E(Q)=\delta_{Q}\left(C_{S}(Q)\right)$.

To see this, fix $\psi \in \operatorname{Mor}_{\mathcal{L}}(P, Q)$ and $g \in C_{S}(Q)$ such that $\delta_{Q}(g) \circ \psi=\psi$. Set $Q_{0}=\pi(\psi)(P)$ and $\psi_{0}=\left.\psi\right|_{P, Q_{0}}$. Thus $\psi=\iota_{Q_{0}}^{Q} \circ \psi_{0}$ where $\psi_{0}$ is an isomorphism, so $\delta_{Q}(g) \circ \iota_{Q_{0}}^{Q}=\iota_{Q_{0}}^{Q}$. In other words, $\delta_{Q_{0}, Q}(g)=\delta_{Q_{0}, Q}(1)$, and so $g=1$ since $\delta_{Q_{0}, Q}$ is injective by (c).

(g) Fix $P \in \operatorname{Ob}(\mathcal{L})$; we claim $P$ is $\mathcal{F}$-quasicentric. It suffices to show this when $P$ is fully centralized. If $P$ is not $\mathcal{F}$-quasicentric, then by definition, there is some $Q$ and some $\operatorname{Id} \neq \alpha \in \operatorname{Aut}_{\mathcal{F}}(Q)$ such that $P \leq Q \leq P C_{S}(P),\left.\alpha\right|_{P}=\operatorname{Id}_{P}$, and $\alpha$ has order prime to $p$. Assume this is the case, and choose $\psi \in \operatorname{Aut}_{\mathcal{L}}(Q)$ such that $\pi(\psi)=\alpha$. We can assume $\psi$ also has order prime to $p$; otherwise replace it by $\psi^{k}$ for some appropriate $k$. Then $\pi\left(\left.\psi\right|_{P, P}\right)=\operatorname{Id}_{P}$, so $\left.\psi\right|_{P, P}=\delta_{P}(g)$ for some $g \in C_{S}(P)$ by axiom (A). Thus $\left.\psi\right|_{P, P}$ has $p$-power order and order prime to $p$, so $\left.\psi\right|_{P, P}=\operatorname{Id}$ in $\operatorname{Aut}_{\mathcal{L}}(P)$. But this means that $\psi \circ \iota_{P}^{Q}=\operatorname{Id}_{Q} \circ \iota_{P}^{Q}$, so $\psi=\operatorname{Id}$ since $\iota_{P}^{Q}$ is an epimorphism by (f). Hence $\alpha=\operatorname{Id}_{Q}$, which contradicts the original assumption on $\alpha$.

Since we want to construct extensions of fusion and linking systems, we must say what we mean by automorphisms of linking systems and by normal linking subsystems. We first look at automorphisms.

Definition 5. For any linking system $\mathcal{L}$, an automorphism of categories $\alpha \in \operatorname{Aut}(\mathcal{L})$ is isotypical if for all $P \in \operatorname{Ob}(\mathcal{L}), \alpha\left(\delta_{P}(P)\right)=\delta_{\alpha(P)}(\alpha(P))$. Let $\operatorname{Aut}_{\text {typ }}^{I}(\mathcal{L})$ be the group of isotypical automorphisms of $\mathcal{L}$ which send inclusions to inclusions.

Let $F: \mathcal{L} \longrightarrow$ Grp be the functor $\pi: \mathcal{L} \longrightarrow \mathcal{F}$ followed by the forgetful functor from $\mathcal{F}$ to groups. When all objects in $\mathcal{L}$ are $\mathcal{F}$-centric, then an automorphism $\alpha$ of a linking system $\mathcal{L}$ is isotypical if and only if there is a natural isomorphism of functors $F \circ \alpha \cong F$. This was shown for centric linking systems in [3, Lemma 8.2], and the same proof applies in this more general setting.

The next proposition shows that each $\alpha \in \operatorname{Aut}_{\text {typ }}^{I}(\mathcal{L})$ induces an automorphism of $\mathcal{F}$ in a natural way.

Proposition 6. Let $\mathcal{L}$ be a linking system associated to a fusion system $\mathcal{F}$ over a p-group $S$, with structure functors $\mathcal{T}_{\mathrm{Ob}(\mathcal{L})}(S) \stackrel{\delta}{\longrightarrow} \mathcal{L} \stackrel{\pi}{\longrightarrow} \mathcal{F}$. Fix $\alpha \in \operatorname{Aut}_{\text {typ }}^{I}(\mathcal{L})$. Let $\beta \in \operatorname{Aut}(S)$ be such that $\alpha\left(\delta_{S}(g)\right)=\delta_{S}(\beta(g))$ for all $g \in S$. Then $\beta$ is "fusion-preserving" in the following sense: there is an automorphism $\widehat{\alpha}$ of the category $\mathcal{F}$ which sends $P \leq S$ to $\beta(P)$ and sends $\varphi \in \operatorname{Hom}_{\mathcal{F}}(P, Q)$ to $\beta \varphi \beta^{-1} \in$ $\operatorname{Hom}_{\mathcal{F}}(\beta(P), \beta(Q))$. Furthermore, $\pi \circ \alpha=\widehat{\alpha} \circ \pi$.

Proof. Clearly, $\alpha(S)=S$, and hence $\alpha$ sends $\delta_{S}(S)$ to itself. Thus $\beta$ is well defined. Since $\alpha$ sends inclusions to inclusions, it commutes with restrictions. So for $P \in$ 
$\operatorname{Ob}(\mathcal{L})$, since $\alpha_{P}$ sends $\delta_{P}(P)$ to $\delta_{\alpha(P)}(\alpha(P)), \alpha_{S}$ sends $\delta_{S}(P)$ to $\delta_{S}(\alpha(P))$, and thus $\alpha(P)=\beta(P)$ since $\delta_{S}$ is a monomorphism (Proposition $4(\mathrm{c})$ ). Furthermore, for each $g \in P, \alpha$ sends $\delta_{P}(g)$ to $\delta_{\beta(P)}(\beta(g))$.

Now fix $P, Q \in \operatorname{Ob}(\mathcal{L})$ and $\psi \in \operatorname{Mor}_{\mathcal{L}}(P, Q)$, and set $\varphi=\pi(\psi) \in \operatorname{Hom}_{\mathcal{F}}(P, Q)$. For each $g \in P, \alpha$ sends

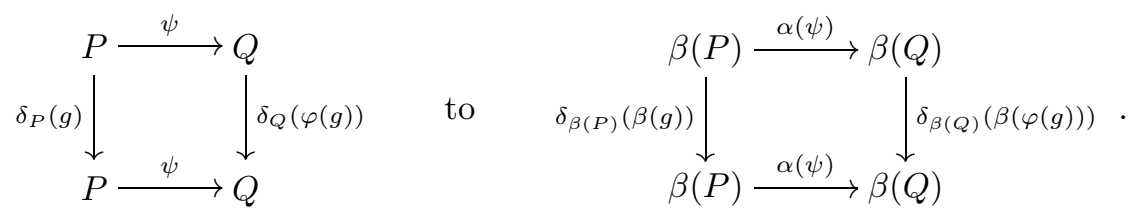

By axiom $(\mathrm{C})$ in Definition 3 these squares commute, and also (since morphisms in $\mathcal{L}$ are epimorphisms) $\beta(\varphi(g))=\pi(\alpha(\psi))(\beta(g))$. Thus

$$
\pi(\alpha(\psi))=\beta \pi(\psi) \beta^{-1} \text {. }
$$

In particular, $\beta \varphi \beta^{-1} \in \operatorname{Hom}_{\mathcal{F}}(\beta(P), \beta(Q))$ for each $P, Q \in \operatorname{Ob}(\mathcal{L})$ and $\varphi \in$ $\operatorname{Hom}_{\mathcal{F}}(P, Q)$. Since $\operatorname{Ob}(\mathcal{L})$ includes all subgroups which are $\mathcal{F}$-centric and $\mathcal{F}$ radical, all morphisms in $\mathcal{F}$ are composites of restrictions of morphisms between objects of $\mathcal{L}$ (cf. [3, Theorem A.10]). Hence there is a well-defined functor $\widehat{\alpha}$ from $\mathcal{F}$ to itself which sends each $P \leq S$ to $\beta(P)$ and sends each $\varphi \in \operatorname{Hom}_{\mathcal{F}}(P, Q)$ to $\beta \varphi \beta^{-1}$. This is an automorphism of the category $\mathcal{F}$ by the same argument applied to $\alpha^{-1}$. By (1), $\pi \circ \alpha=\widehat{\alpha} \circ \pi$.

The following definition of a normal fusion subsystem is the most convenient for our purposes. When $\mathcal{F}$ is a fusion system over $S$ and $S_{0} \unlhd S$, then $S_{0}$ is strongly closed if no element of $S_{0}$ is $\mathcal{F}$-conjugate to any element of $S \backslash S_{0}$.

Definition 7. Let $\mathcal{F}$ be a saturated fusion system over a $p$-group $S$, and let $\mathcal{F}_{0} \subseteq \mathcal{F}$ be a subcategory which is a saturated fusion subsystem over a subgroup $S_{0} \leq S$. Then $\mathcal{F}_{0}$ is normal in $\mathcal{F}\left(\mathcal{F}_{0} \unlhd \mathcal{F}\right)$ if

(i) $S_{0}$ is strongly closed in $\mathcal{F}$;

(ii) for all $P, Q \leq S_{0}$ and $\varphi \in \operatorname{Hom}_{\mathcal{F}}(P, Q)$, there are morphisms $\alpha \in \operatorname{Aut}_{\mathcal{F}}\left(S_{0}\right)$ and $\varphi_{0} \in \operatorname{Hom}_{\mathcal{F}_{0}}(\alpha(P), Q)$ such that $\varphi=\left.\varphi_{0} \circ \alpha\right|_{P, \alpha(P)}$;

(iii) for each $P, Q \leq S_{0}$, each $\varphi \in \operatorname{Hom}_{\mathcal{F}_{0}}(P, Q)$, and each $\beta \in \operatorname{Aut}_{\mathcal{F}}\left(S_{0}\right), \beta \varphi \beta^{-1} \in$ $\operatorname{Hom}_{\mathcal{F}_{0}}(\beta(P), \beta(Q))$.

This is equivalent to Puig's definition of a normal subsystem [10, $\S 6.4]$, and also to Aschbacher's definition [2, §3] of an $\mathcal{F}$-invariant subsystem, except that they do not require the subsystem to be saturated. See [2, Theorem 3.3] for a proof of the equivalence of these definitions.

For example, when $G_{0} \unlhd G$ are finite groups, $S \in \operatorname{Syl}_{p}(G)$, and $S_{0}=S \cap G_{0} \in$ $\operatorname{Syl}_{p}\left(G_{0}\right)$, then $\mathcal{F}_{S_{0}}\left(G_{0}\right)$ is a normal subsystem of $\mathcal{F}_{S}(G)$ under the above definition. The first and last conditions clearly hold, and (ii) holds by the Frattini argument: $G=G_{0} \cdot N_{G}\left(S_{0}\right)$, since any subgroup $G$-conjugate to $S_{0}$ is also $G_{0}$-conjugate.

There is now an obvious analogous definition of a normal linking subsystem.

Definition 8. Fix a pair of saturated fusion systems $\mathcal{F}_{0} \unlhd \mathcal{F}$ over $p$-groups $S_{0} \unlhd S$ such that $\mathcal{F}_{0}$ is normal in $\mathcal{F}$, and let $\mathcal{L}_{0} \subseteq \mathcal{L}$ be associated linking systems. Then $\mathcal{L}_{0}$ is normal in $\mathcal{L}\left(\mathcal{L}_{0} \unlhd \mathcal{L}\right)$ if

(i) $\operatorname{Ob}(\mathcal{L})=\left\{P \leq S \mid P \cap S_{0} \in \mathrm{Ob}\left(\mathcal{L}_{0}\right)\right\}$; 
(ii) for all $P, Q \in \operatorname{Ob}\left(\mathcal{L}_{0}\right)$ and $\psi \in \operatorname{Mor}_{\mathcal{L}}(P, Q)$, there are morphisms $\gamma \in$ $\operatorname{Aut}_{\mathcal{L}}\left(S_{0}\right)$ and $\psi_{0} \in \operatorname{Mor}_{\mathcal{L}_{0}}(\gamma(P), Q)$ such that $\psi=\left.\psi_{0} \circ \gamma\right|_{P, \gamma(P)} ;$

(iii) for all $\gamma \in \operatorname{Aut}_{\mathcal{L}}\left(S_{0}\right), P, Q \in \operatorname{Ob}\left(\mathcal{L}_{0}\right)$, and $\psi \in \operatorname{Mor}_{\mathcal{L}_{0}}(P, Q),\left.\gamma\right|_{Q, \gamma(Q)} \circ \psi \circ$ $\left.\gamma\right|_{P, \gamma(P)} ^{-1}$ is in $\operatorname{Mor}_{\mathcal{L}_{0}}(\gamma(P), \gamma(Q))$.

Here, in (ii) and (iii), we write $\gamma(P)=\pi(\gamma)(P)$ and $\gamma(Q)=\pi(\gamma)(Q)$ for short.

In fact, condition (ii) in Definition 8 follows from the other conditions in that definition, together with condition (ii) in Definition 7 But we include it here anyway to make the analogy between the two definitions clearer.

Whenever $\mathcal{L}_{0} \unlhd \mathcal{L}$ is a pair of linking systems over $p$-groups $S_{0} \unlhd S$, then the geometric realization $\left|\mathcal{L}_{0}\right|$ has the homotopy type of a covering space over $|\mathcal{L}|$ with covering group the quotient $\operatorname{Aut}_{\mathcal{L}}\left(S_{0}\right) / \operatorname{Aut}_{\mathcal{L}_{0}}\left(S_{0}\right)$. We don't prove this here since it isn't used, but it follows by essentially the same proof as that of [5, Theorem 3.9] or [8, Proposition 4.1(d)].

We are now ready to describe the procedure for constructing extensions of linking systems. To help motivate the hypotheses, we first describe an analogous construction with groups. Assume we are given three groups $H_{0}, H$, and $G_{0}$, where $H_{0} \leq G_{0}$ and $H_{0} \unlhd H$, together with an action $\tau$ of $H$ on $G_{0}$ which leaves $H_{0}$ invariant. Regard $\tau$ as a homomorphism from $H$ to $\operatorname{Aut}\left(G_{0}: H_{0}\right)$, where $\operatorname{Aut}\left(G_{0}: H_{0}\right)$ is the group of automorphisms of $G_{0}$ which leave $H_{0}$ invariant. We want to construct a group $G=G_{0} H$, where $G_{0} \unlhd G$, and where the conjugation action of $H$ on $G_{0}$ is that defined by $\tau$. The obvious way to do this is to start with the semidirect product $G_{0} \rtimes H$ defined by $\tau$, and then set $G=\left(G_{0} \rtimes H\right) /\left\{\left(g, g^{-1}\right) \mid g \in H_{0}\right\}$. In order to do this, the set $\left\{\left(g, g^{-1}\right)\right\}$ must be a normal subgroup, and one quickly discovers that the necessary and sufficient condition for this to be the case is for the following diagram to commute:

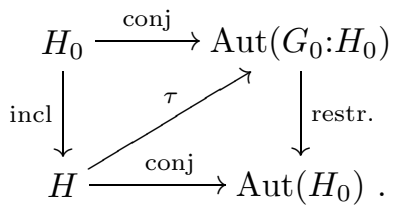

The hypotheses in Theorem 9 are similar, except that $G_{0} \unlhd G$ are replaced by a pair of linking systems $\mathcal{L}_{0} \unlhd \mathcal{L}$.

The following theorem generalizes [5, Theorem 4.6] and also generalizes a related result in 6. Recall that by definition, the set of objects in a linking system $\mathcal{L}$ associated to a fusion system $\mathcal{F}$ over $S$ is closed under $\mathcal{F}$-conjugacy and overgroups and must contain among its objects all subgroups which are $\mathcal{F}$-centric and $\mathcal{F}$ radical. By the conjugation action of $\psi \in \operatorname{Aut}_{\mathcal{L}}(S)$ on $\mathcal{L}$ is meant the action which sends $P \leq S$ to $\pi(\psi)(P)$, and which sends $\alpha \in \operatorname{Mor}(\mathcal{L})$ to $\psi \alpha \psi^{-1}$ (after replacing each $\psi$ by the appropriate restriction).

Theorem 9. Fix a saturated fusion system $\mathcal{F}_{0}$ over a finite p-group $S_{0}$, and let $\mathcal{L}_{0}$ be a linking system associated to $\mathcal{F}_{0}$. Set $\mathcal{H}_{0}=\operatorname{Ob}\left(\mathcal{L}_{0}\right)$. Set $\Gamma_{0}=\operatorname{Aut}_{\mathcal{L}_{0}}\left(S_{0}\right)$, and regard $S_{0}$ as a subgroup of $\Gamma_{0}$ via the inclusion of $\mathcal{T}_{\mathcal{H}_{0}}\left(S_{0}\right)$ into $\mathcal{L}_{0}$. Thus $S_{0}=O_{p}\left(\Gamma_{0}\right)$, since $\Gamma_{0} / S_{0}$ has order prime to $p$ by Proposition $4(d)$. Fix a finite group $\Gamma$ such that $\Gamma_{0} \unlhd \Gamma$, and a homomorphism $\tau: \Gamma \longrightarrow \operatorname{Aut}_{\text {typ }}^{I}\left(\mathcal{L}_{0}\right)$ which 
makes both triangles in the following diagram commute:

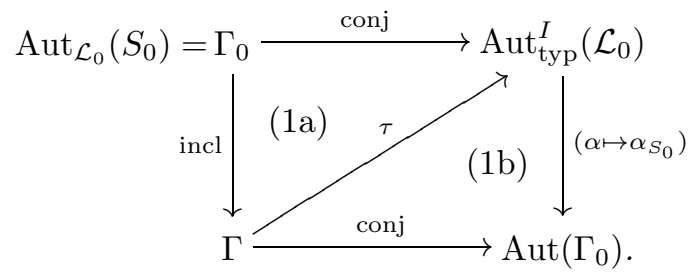

Let $\mathcal{F}_{1}$ be the smallest fusion system over $S_{0}$ (not necessarily saturated) such that $\mathcal{F}_{1} \supseteq \mathcal{F}_{0}$ and $\operatorname{Aut}_{\mathcal{F}_{1}}\left(S_{0}\right) \geq \operatorname{Aut}_{\Gamma}\left(S_{0}\right)$, where $\Gamma$ acts on $S_{0}=O_{p}\left(\Gamma_{0}\right) \unlhd \Gamma$ via conjugation. Fix $S \in \operatorname{Syl}_{p}(\Gamma)$. Then there is a saturated fusion system $\mathcal{F}$ over $S$ which contains $\mathcal{F}_{1}$ as a full subcategory, and such that $\mathcal{F}_{0} \unlhd \mathcal{F}$.

Assume, in addition, that

$$
C_{\Gamma}\left(S_{0}\right) \text { is a p-group, }
$$

and also that

$$
\Gamma / \Gamma_{0} \text { is a p-group, or each } P \in \mathcal{H}_{0} \text { is } \mathcal{F}_{0} \text {-centric, }
$$

or more generally,

$$
\begin{aligned}
P \in \mathcal{H}_{0}, P \leq Q \leq P \cdot C_{S_{0}}(P), \alpha \in \operatorname{Aut}_{\mathcal{F}_{1}}(Q), & \left.\alpha\right|_{P}=\operatorname{Id}_{P} \\
& \Longrightarrow \alpha \text { has p-power order. }
\end{aligned}
$$

Then $\mathcal{F}$ can be chosen so as to have an associated linking system $\mathcal{L}$ for which $\mathcal{L}_{0} \unlhd \mathcal{L}$,

$$
\mathrm{Ob}(\mathcal{L})=\mathcal{H} \stackrel{\text { def }}{=}\left\{P \leq S \mid P \cap S_{0} \in \mathcal{H}_{0}\right\}
$$

and $\operatorname{Aut}_{\mathcal{L}}\left(S_{0}\right)=\Gamma$ with the action on $\mathcal{L}_{0}$ given by $\tau$. If $\mathcal{L}^{\prime}$ is another linking system, associated to a saturated fusion system $\mathcal{F}^{\prime}$ over $S$, such that $\mathcal{L}_{0} \unlhd \mathcal{L}^{\prime}, \mathcal{F}_{0} \unlhd \mathcal{F}^{\prime}$, $\operatorname{Ob}\left(\mathcal{L}^{\prime}\right)=\mathcal{H}$, and $\operatorname{Aut}_{\mathcal{L}^{\prime}}\left(S_{0}\right)=\Gamma$ with the action on $\mathcal{L}_{0}$ given by $\tau$ and the given inclusion $S \leq \Gamma$, then $\mathcal{F}^{\prime}=\mathcal{F}$ and $\mathcal{L}^{\prime} \cong \mathcal{L}$.

Proof. The categories $\mathcal{L}$ and $\mathcal{F}$ will be constructed in Steps 1 and 2. We then show $\mathcal{F}$ is $\mathcal{H}$-saturated in Steps 3 and 4 and finish the proof that $\mathcal{F}$ is saturated in Step 5 . Step 5 is essentially a corrected version of Step 4 in the proof of 5 , Theorem 4.6]. We prove (31) implies (3) at the beginning of Step 6. Afterwards, we assume (2) and (3) throughout the rest of the proof, show $\mathcal{L}$ is a linking system in Steps 6 and 7 , and prove its uniqueness in Step 8. The normality of $\mathcal{F}_{0}$ in $\mathcal{F}$ and of $\mathcal{L}_{0}$ in $\mathcal{L}$ are shown at the end of Steps 5 and 7 , respectively.

We first fix some notation. For all $P \leq S$, we write $P_{0}=P \cap S_{0}$. Let

$$
\mathcal{T}_{\mathcal{H}_{0}}\left(S_{0}\right) \stackrel{\delta_{0}}{\longrightarrow} \mathcal{L}_{0} \stackrel{\pi_{0}}{\longrightarrow} \mathcal{F}_{0}
$$

be the structure functors for the linking system $\mathcal{L}_{0}$. For $P \leq Q$, set $\iota_{P}^{Q}=\left(\delta_{0}\right)_{P, Q}(1)$. Recall that we regard $S_{0}$ as a subgroup of $\Gamma_{0}=\operatorname{Aut}_{\mathcal{L}_{0}}\left(S_{0}\right)$ via $\left(\delta_{0}\right)_{S_{0}}$.

For each $\gamma \in \Gamma$, let $c_{\gamma} \in \operatorname{Aut}\left(S_{0}\right)$ denote conjugation by $\gamma$ on $S_{0}=O_{p}\left(\Gamma_{0}\right) \unlhd \Gamma$. By the commutativity of (1b), this is the restriction to $S_{0}$ of $\tau(\gamma)_{S_{0}} \in \operatorname{Aut}\left(\Gamma_{0}\right)$. Hence by Proposition 6, $c_{\gamma}$ is fusion-preserving (induces an automorphism of the category $\left.\mathcal{F}_{0}\right)$, and $\tau(\gamma)(P)=c_{\gamma}(P)$ for all $P \in \mathcal{H}_{0}$. To simplify notation below, we write $\gamma(P)=\tau(\gamma)(P)$ to denote this action of $\gamma$ on $\mathcal{H}_{0}$. 
For each $\gamma_{0} \in \Gamma_{0}=\operatorname{Aut}_{\mathcal{L}_{0}}\left(S_{0}\right), \gamma_{0}$ acts on the set $\operatorname{Mor}\left(\mathcal{L}_{0}\right)$ by composing on the left or right with $\gamma_{0}$ and its restrictions. Thus for any $\varphi \in \operatorname{Mor}_{\mathcal{L}_{0}}(P, Q)$, we set

$$
\gamma_{0} \varphi=\left.\gamma_{0}\right|_{Q, \gamma_{0}(Q)} \circ \varphi \in \operatorname{Mor}_{\mathcal{L}_{0}}\left(P, \gamma_{0}(Q)\right)
$$

and

$$
\varphi \gamma_{0}=\left.\varphi \circ \gamma_{0}\right|_{\gamma_{0}^{-1}(P), P} \in \operatorname{Mor}_{\mathcal{L}_{0}}\left(\gamma_{0}^{-1}(P), Q\right) .
$$

Here, we write $\gamma_{0}(P)=\pi\left(\gamma_{0}\right)(P)$ for short. This defines natural left and right actions of $\Gamma_{0}$ on the set $\operatorname{Mor}\left(\mathcal{L}_{0}\right)$. By the commutativity of $(1 \mathrm{a})$, the conjugation action $\psi \mapsto \gamma_{0} \psi \gamma_{0}^{-1}$ on $\operatorname{Mor}\left(\mathcal{L}_{0}\right)$ is the restriction to $\Gamma_{0}$ of $\tau$; in particular, $\gamma_{0}(P)=$ $\tau\left(\gamma_{0}\right)(P)$ as in the last paragraph.

Step 1 . We first define categories $\mathcal{L}_{1} \supseteq \mathcal{L}_{0}$ and $\mathcal{F}_{1} \supseteq \mathcal{F}_{0}$, where $\operatorname{Ob}\left(\mathcal{F}_{1}\right)=\operatorname{Ob}\left(\mathcal{F}_{0}\right)$ and $\operatorname{Ob}\left(\mathcal{L}_{1}\right)=\mathcal{H}_{0}$. Set

$$
\operatorname{Mor}\left(\mathcal{L}_{1}\right)=\operatorname{Mor}\left(\mathcal{L}_{0}\right) \times_{\Gamma_{0}} \Gamma=\left(\operatorname{Mor}\left(\mathcal{L}_{0}\right) \times \Gamma\right) / \sim,
$$

where $(\varphi, \gamma) \sim\left(\varphi^{\prime}, \gamma^{\prime}\right)$ if and only if there is $\gamma_{0} \in \Gamma_{0}$ such that $\varphi^{\prime}=\varphi \gamma_{0}$ and $\gamma^{\prime}=\gamma_{0}^{-1} \gamma$. Thus $\left(\varphi \gamma_{0}, \gamma\right) \sim\left(\varphi, \gamma_{0} \gamma\right)$ for all $\varphi \in \operatorname{Mor}\left(\mathcal{L}_{0}\right), \gamma_{0} \in \Gamma_{0}$, and $\gamma \in \Gamma$. If $\varphi \in \operatorname{Mor}_{\mathcal{L}_{0}}(P, Q)$, then $\llbracket \varphi, \gamma \rrbracket \in \operatorname{Mor}_{\mathcal{L}_{1}}\left(\gamma^{-1}(P), Q\right)$ denotes the equivalence class of the pair $(\varphi, \gamma)$. Composition is defined by

$$
\llbracket \psi, \eta \rrbracket \circ \llbracket \varphi, \gamma \rrbracket=\llbracket \psi \circ \tau(\eta)(\varphi), \eta \gamma \rrbracket .
$$

Here, $\tau(\eta)(\varphi) \in \operatorname{Mor}_{\mathcal{L}_{0}}(\eta(P), \eta(Q))$ for $\varphi \in \operatorname{Mor}_{\mathcal{L}_{0}}(P, Q)($ recall $\eta(P)=\tau(\eta)(P)$, etc.).

To show composition is well defined, we note that for all $\psi, \varphi \in \operatorname{Mor}\left(\mathcal{L}_{0}\right)$ with appropriate domain and range, and all $\eta_{0}, \gamma_{0} \in \Gamma_{0}$, and $\eta, \gamma \in \Gamma$,

$$
\begin{aligned}
& \llbracket \psi \eta_{0}, \eta \rrbracket \circ \llbracket \varphi \gamma_{0}, \gamma \rrbracket=\llbracket \psi \eta_{0} \circ \tau(\eta)\left(\varphi \gamma_{0}\right), \eta \gamma \rrbracket=\llbracket \psi \eta_{0} \circ \tau(\eta)(\varphi),\left(\eta \gamma_{0} \eta^{-1}\right) \eta \gamma \rrbracket \\
& \quad=\llbracket \psi \eta_{0} \circ \tau(\eta)(\varphi) \eta_{0}^{-1}, \eta_{0} \eta \gamma_{0} \gamma \rrbracket=\llbracket \psi \circ \tau\left(\eta_{0} \eta\right)(\varphi), \eta_{0} \eta \gamma_{0} \gamma \rrbracket=\llbracket \psi, \eta_{0} \eta \rrbracket \circ \llbracket \varphi, \gamma_{0} \gamma \rrbracket .
\end{aligned}
$$

The second equality follows from the commutativity of (1b), and the fourth follows from that of (1a).

We claim that

$$
\text { all morphisms in } \mathcal{L}_{1} \text { are monomorphisms and epimorphisms. }
$$

For any $\llbracket \varphi, \gamma \rrbracket, \llbracket \varphi^{\prime}, \gamma^{\prime} \rrbracket$, and $\llbracket \psi, \eta \rrbracket$ with appropriate domain and range,

$$
\begin{gathered}
\llbracket \psi, \eta \rrbracket \circ \llbracket \varphi, \gamma \rrbracket=\llbracket \psi, \eta \rrbracket \circ \llbracket \varphi^{\prime}, \gamma^{\prime} \rrbracket \Longrightarrow \llbracket \psi \circ \tau(\eta)(\varphi), \eta \gamma \rrbracket=\llbracket \psi \circ \tau(\eta)\left(\varphi^{\prime}\right), \eta \gamma^{\prime} \rrbracket \\
\Longrightarrow \exists \gamma_{0} \in \Gamma_{0}, \eta \gamma=\gamma_{0}^{-1} \eta \gamma^{\prime} \text { and } \psi \circ \tau(\eta)(\varphi)=\psi \circ \tau(\eta)\left(\varphi^{\prime}\right) \circ \gamma_{0} \\
\Longrightarrow \gamma=\left(\eta^{-1} \gamma_{0} \eta\right)^{-1} \gamma^{\prime} \text { and } \varphi=\varphi^{\prime} \circ \tau\left(\eta^{-1}\right)\left(\gamma_{0}\right)
\end{gathered}
$$

since morphisms in $\mathcal{L}_{0}$ are monomorphisms (Proposition $4(\mathrm{f})$ ). Also, $\tau\left(\eta^{-1}\right)\left(\gamma_{0}\right)=$ $\eta^{-1} \gamma_{0} \eta$ by the commutativity of $(1 \mathrm{~b})$, so $\llbracket \varphi, \gamma \rrbracket=\llbracket \varphi^{\prime}, \gamma^{\prime} \rrbracket$, and hence $\llbracket \psi, \eta \rrbracket$ is a monomorphism. The proof that morphisms are epimorphisms is similar.

Set $\operatorname{Aut}_{\Gamma}\left(S_{0}\right)=\left\{c_{\gamma} \in \operatorname{Aut}\left(S_{0}\right) \mid \gamma \in \Gamma\right\}$. Let $\mathcal{F}_{1}$ be the smallest fusion system over $S_{0}$ which contains $\mathcal{F}_{0}$ and $\operatorname{Aut}_{\Gamma}\left(S_{0}\right)$. Define

$$
\pi_{1}: \mathcal{L}_{1} \longrightarrow \mathcal{F}_{1}
$$


by setting $\pi_{1}(\llbracket \varphi, \gamma \rrbracket)=\pi_{0}(\varphi) \circ c_{\gamma}$. To show this is a functor (that it preserves composition), we must show that the following square commutes:

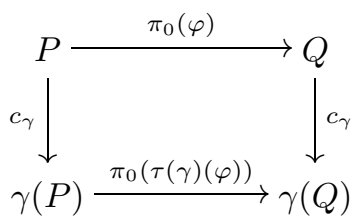

for each $\varphi \in \operatorname{Mor}_{\mathcal{L}_{0}}(P, Q)$ and each $\gamma \in \Gamma$; this follows from the commutativity of (1b) together with the last statement in Proposition 6 (applied with $\alpha=\tau(\gamma)$ and $\left.\beta=c_{\gamma}\right)$. Since $\pi_{1}\left(\mathcal{L}_{1}\right)$ contains $\left.\mathcal{F}_{0}\right|_{\mathcal{H}_{0}}$ and $\operatorname{Aut}_{\Gamma}\left(S_{0}\right)$, and is closed under restrictions of morphisms to subgroups in $\mathcal{H}_{0}$ (Proposition 4 (b)), $\pi_{1}$ maps onto $\left.\mathcal{F}_{1}\right|_{\mathcal{H}_{0}}$.

We regard $\mathcal{L}_{0}$ as a subcategory of $\mathcal{L}_{1}$ by identifying $\varphi \in \operatorname{Mor}_{\mathcal{L}_{0}}(P, Q)$ with $\llbracket \varphi, 1 \rrbracket \in \operatorname{Mor}_{\mathcal{L}_{1}}(P, Q)$. By construction, $\pi_{0}=\left.\pi_{1}\right|_{\mathcal{L}_{0}}$. For $P \leq Q$ in $\mathcal{H}_{0}$, the inclusion morphism $\iota_{P}^{Q}=\left(\delta_{0}\right)_{P, Q}(1)$ for $\mathcal{L}_{0}$ is also considered as an inclusion morphism in $\mathcal{L}_{1}$. The existence of restriction morphisms in $\mathcal{L}_{0}$ (Proposition 4(b)) carries over easily to the existence of restriction morphisms in $\mathcal{L}_{1}$, and they are unique by (4).

For all $P, Q \in \mathcal{H}_{0}$, define

$$
\delta_{P, Q}: N_{S}(P, Q) \longrightarrow \operatorname{Mor}_{\mathcal{L}_{1}}(P, Q)
$$

by setting $\delta_{P, Q}(s)=\llbracket \iota_{s(P)}^{Q}, s \rrbracket$. When $s \in S_{0}, \llbracket \iota_{s(P)}^{Q}, s \rrbracket=\llbracket\left(\delta_{0}\right)_{P, Q}(s), 1 \rrbracket ;$ thus $\delta_{P, Q}$ extends the monomorphism $\left(\delta_{0}\right)_{P, Q}$ from $N_{S_{0}}(P, Q)$ to $\operatorname{Mor}_{\mathcal{L}_{0}}(P, Q)$ defined for $\mathcal{L}_{0}$. To simplify the notation, we write $\delta(x)=\delta_{P, Q}(x)$ when $P$ and $Q$ are understood.

We claim that for all $P, Q \in \mathcal{H}_{0}, \psi \in \operatorname{Mor}_{\mathcal{L}_{1}}(P, Q)$, and $x \in P$,

$$
\delta\left(\pi_{1}(\psi)(x)\right) \circ \psi=\psi \circ \delta(x) .
$$

Set $\psi=\llbracket \varphi, \gamma \rrbracket$, where $\gamma \in \Gamma$ and $\varphi \in \operatorname{Mor}_{\mathcal{L}_{0}}(\gamma(P), Q)$. Then

$$
\begin{aligned}
\psi \circ \delta(x) & =\llbracket \varphi, \gamma \rrbracket \circ \llbracket \operatorname{Id}_{P}, x \rrbracket=\llbracket \varphi, \gamma x \rrbracket=\llbracket \varphi, c_{\gamma}(x) \gamma \rrbracket=\llbracket \varphi \circ \delta_{0}\left(c_{\gamma}(x)\right), \gamma \rrbracket \\
& =\llbracket \delta_{0}\left(\pi_{0}(\varphi)\left(c_{\gamma}(x)\right)\right) \circ \varphi, \gamma \rrbracket=\llbracket \delta_{0}\left(\pi_{1}(\psi)(x)\right) \circ \varphi, \gamma \rrbracket \\
& =\llbracket \delta_{0}\left(\pi_{1}(\psi)(x)\right), 1 \rrbracket \circ \llbracket \varphi, \gamma \rrbracket=\delta\left(\pi_{1}(\psi)(x)\right) \circ \psi,
\end{aligned}
$$

where the fifth equality holds by axiom $(\mathrm{C})$ for the linking system $\mathcal{L}_{0}$.

We next show that morphisms in $\mathcal{L}_{1}$ have the following extension property:

$$
\forall P, Q \in \mathcal{H}_{0}, \psi \in \operatorname{Iso}_{\mathcal{L}_{1}}(P, Q) \text {, and } \bar{P}, \bar{Q} \leq S_{0} \text { for which } P \unlhd \bar{P}, Q \unlhd \bar{Q},
$$$$
\text { and } \psi \delta(\bar{P}) \psi^{-1} \leq \delta(\bar{Q}), \quad \exists ! \bar{\psi} \in \operatorname{Mor}_{\mathcal{L}_{1}}(\bar{P}, \bar{Q}) \text { such that }\left.\bar{\psi}\right|_{P, Q}=\psi \text {. }
$$

Set $\psi=\llbracket \varphi, \gamma \rrbracket$, where $\varphi \in \operatorname{Mor}_{\mathcal{L}_{0}}(\gamma(P), Q)$. For all $x \in \bar{P}$,

$\llbracket \varphi, \gamma \rrbracket \circ \llbracket \delta_{0}(x), 1 \rrbracket \circ \llbracket \varphi, \gamma \rrbracket^{-1}=\llbracket \varphi \circ \tau(\gamma)\left(\delta_{0}(x)\right) \circ \varphi^{-1}, 1 \rrbracket=\llbracket \varphi \circ \delta_{0}\left(c_{\gamma}(x)\right) \circ \varphi^{-1}, 1 \rrbracket \in \delta(\bar{Q})$,

where $\tau(\gamma)\left(\delta_{0}(x)\right)=\delta_{0}\left(c_{\gamma}(x)\right)$ by the commutativity of $(1 \mathrm{~b})$. Thus $\varphi \delta_{0}(\gamma(\bar{P})) \varphi^{-1} \leq$ $\delta_{0}(\bar{Q})$, so $\varphi$ extends to $\bar{\varphi} \in \operatorname{Mor}_{\mathcal{L}_{0}}(\gamma(\bar{P}), \bar{Q})$ by Proposition $4(\mathrm{e})$. Set $\bar{\psi}=\llbracket \bar{\varphi}, \gamma \rrbracket$. Then $\left.\bar{\psi}\right|_{P, Q}=\psi$ since $\tau(\gamma)\left(\iota_{P}^{\bar{P}}\right)=\iota_{\gamma(P)}^{\gamma(\bar{P})}(\tau(\gamma)$ sends inclusions to inclusions), and this proves (5).

Step 2. We next construct categories $\mathcal{L}$ and $\mathcal{F}_{2}$, both of which have object sets $\mathcal{H}$, and which contain $\mathcal{L}_{1}$ and the restriction of $\mathcal{F}_{1}$ to $\mathcal{H}_{0}$, respectively. Afterwards, we let $\mathcal{F}$ be the fusion system over $S$ generated by $\mathcal{F}_{2}$ and restrictions of morphisms. 
Now let $\mathcal{L}$ be the category with $\operatorname{Ob}(\mathcal{L})=\mathcal{H}$, and where for all $P, Q \in \mathcal{H}$, $\operatorname{Mor}_{\mathcal{L}}(P, Q)=\left\{\psi \in \operatorname{Mor}_{\mathcal{L}_{1}}\left(P_{0}, Q_{0}\right) \mid \forall x \in P \exists y \in Q\right.$ such that $\left.\psi \circ \delta(x)=\delta(y) \circ \psi\right\}$. Let

$$
\begin{aligned}
& \delta_{P, Q}: N_{S}(P, Q) \longrightarrow \operatorname{Mor}_{\mathcal{L}}(P, Q) \\
& \subseteq N_{S}\left(P_{0}, Q_{0}\right) \quad \subseteq \operatorname{Mor}_{\mathcal{L}_{1}}\left(P_{0}, Q_{0}\right)
\end{aligned}
$$

be the restriction of $\delta_{P_{0}, Q_{0}}$. Let $\mathcal{F}_{2}$ be the category with $\mathrm{Ob}\left(\mathcal{F}_{2}\right)=\mathcal{H}$, and where

$$
\begin{aligned}
\operatorname{Mor}_{\mathcal{F}_{2}}(P, Q)=\left\{\varphi \in \operatorname{Hom}(P, Q) \mid \exists \psi \in \operatorname{Mor}_{\mathcal{L}_{1}}\left(P_{0}, Q_{0}\right)\right. & \\
& \text { where } \psi \circ \delta(x)=\delta(\varphi(x)) \circ \psi \forall x \in P\} .
\end{aligned}
$$

Let $\pi: \mathcal{L} \longrightarrow \mathcal{F}_{2}$ be the functor which is the the identity on objects, and which sends $\psi \in \operatorname{Mor}_{\mathcal{L}}(P, Q)$ to the homomorphism $\pi(\psi)(x)=y$ if $\psi \circ \delta(x)=\delta(y) \circ \psi$ (uniquely defined by (4)). This is clearly a functor: it is seen to preserve composition by juxtaposing the commutative squares which define $\pi$ on morphisms.

Let $\mathcal{F}$ be the fusion system over $S$ generated by $\mathcal{F}_{2}$ and restriction of homomorphisms. Since $\mathcal{H}=\operatorname{Ob}\left(\mathcal{F}_{2}\right)$ is closed under overgroups, $\mathcal{F}_{2}$ is a full subcategory of $\mathcal{F}$. Since $\mathcal{L}_{1}$ is a full subcategory of $\mathcal{L}, \operatorname{Hom}_{\mathcal{F}_{1}}(P, Q)=\operatorname{Hom}_{\mathcal{F}_{2}}(P, Q)$ for all $P, Q \in \mathcal{H}_{0}$. If $P, Q \leq S_{0}$ are any subgroups and $\varphi \in \operatorname{Hom}_{\mathcal{F}}(P, Q)$, then $\varphi$ is a composite of restrictions of morphisms in $\mathcal{F}_{2}$, and hence (since $P \in \operatorname{Ob}\left(\mathcal{F}_{2}\right)=\mathcal{H}$ implies $P_{0} \in \mathcal{H}_{0}$ ) a composite of restrictions of morphisms in $\mathcal{F}_{2}$ (equivalently $\mathcal{F}_{1}$ ) between subgroups in $\mathcal{H}_{0}$. Thus $\varphi \in \operatorname{Hom}_{\mathcal{F}_{1}}(P, Q)$, and we conclude that $\mathcal{F}_{1}$ is also a full subcategory of $\mathcal{F}$.

Step 3. We next prove that

each $P \in \mathcal{H}$ is $\mathcal{F}$-conjugate to some $P^{\prime} \in \mathcal{H}$

$$
\text { such that } \delta\left(N_{S}\left(P_{0}^{\prime}\right)\right) \in \operatorname{Syl}_{p}\left(\operatorname{Aut}_{\mathcal{L}}\left(P_{0}^{\prime}\right)\right) \text {. }
$$

Let $\mathcal{P}_{\text {fn }}$ be the set of all $S_{0}$-conjugacy classes $\left[P_{0}^{\prime}\right]$ of subgroups $P_{0}^{\prime} \leq S_{0}$ which are $\mathcal{F}_{0}$-conjugate to $P_{0}$ and fully normalized in $\mathcal{F}_{0}$. (If $P_{0}^{\prime}$ is fully normalized in $\mathcal{F}_{0}$, then so is every subgroup in $\left[P_{0}^{\prime}\right]$.) If $\gamma \in \Gamma$ and $Q_{0}, Q_{0}^{\prime} \in \mathcal{H}_{0}$, then since $\gamma$ acts on $\mathcal{L}_{0}$ and hence on $\mathcal{F}_{0}$ as a group of automorphisms (Proposition 6), $Q_{0}$ is $\mathcal{F}_{0}$-conjugate to $Q_{0}^{\prime}$ if and only if $\gamma\left(Q_{0}\right)$ is $\mathcal{F}_{0}$-conjugate to $\gamma\left(Q_{0}^{\prime}\right)$.

Let $\Gamma^{\prime} \subseteq \Gamma$ be the subset of those $\gamma \in \Gamma$ such that $\gamma\left(P_{0}\right)$ is $\mathcal{F}_{0}$-conjugate to $P_{0}$. For $\gamma_{1}, \gamma_{2} \in \Gamma^{\prime}, \gamma_{1} \gamma_{2}\left(P_{0}\right)$ is $\mathcal{F}_{0}$-conjugate to $\gamma_{1}\left(P_{0}\right)$ since $\gamma_{2}\left(P_{0}\right)$ is $\mathcal{F}_{0}$-conjugate to $P_{0}$, and hence $\gamma_{1} \gamma_{2} \in \Gamma^{\prime}$. Thus $\Gamma^{\prime}$ is a subgroup of $\Gamma$. Since $S_{0} \unlhd \Gamma, Q_{0}$ and $Q_{0}^{\prime}$ are $S_{0}$-conjugate if and only if $\gamma\left(Q_{0}\right)$ and $\gamma\left(Q_{0}^{\prime}\right)$ are. Since each $\gamma \in \Gamma$ acts on $S_{0}$ via the fusion-preserving automorphism $c_{\gamma} \in \operatorname{Aut}\left(S_{0}\right)$ as shown above, $\gamma$ permutes the subgroups fully normalized in $S_{0}$. This proves that $\Gamma^{\prime}$ permutes the set $\mathcal{P}_{\mathrm{fn}}$.

Fix $S^{*} \in \operatorname{Syl}_{p}\left(\Gamma^{\prime}\right)$. Let $\eta \in \Gamma$ be such that $S^{\prime} \stackrel{\text { def }}{=} \eta S^{*} \eta^{-1} \leq S$. Since $\mathcal{P}_{\mathrm{fn}}$ has order prime to $p$ by [5. Proposition 1.16], there is some $\left[P_{0}^{*}\right] \in \mathcal{P}_{\text {fn }}$ fixed by $S^{*}$. In other words, for each $\gamma \in S^{*}, \gamma\left(P_{0}^{*}\right)$ is $S_{0}$-conjugate to $P_{0}^{*}$. So for each $s=\eta \gamma \eta^{-1} \in S^{\prime}$ (where $\left.\gamma \in S^{*}\right), s\left(\eta\left(P_{0}^{*}\right)\right)$ is $S_{0}$-conjugate to $\eta\left(P_{0}^{*}\right)$. Set $Q_{0}=\eta\left(P_{0}^{*}\right)$. Then each coset in $S^{\prime} / S_{0}$ contains some element $s$ which normalizes $Q_{0}$, and hence

$$
\left|N_{S}\left(Q_{0}\right)\right| \geq\left|N_{S_{0}}\left(Q_{0}\right)\right| \cdot\left|S^{\prime} / S_{0}\right|=\left|N_{S_{0}}\left(P_{0}^{*}\right)\right| \cdot\left|S^{*} / S_{0}\right| .
$$

Since $\Gamma^{\prime}$ is the subgroup of elements of $\Gamma$ which send $P_{0}$ to a subgroup in its $\mathcal{F}_{0}$-conjugacy class,

$$
\left|\operatorname{Aut}_{\mathcal{L}}\left(Q_{0}\right)\right|=\left|\operatorname{Aut}_{\mathcal{L}}\left(P_{0}\right)\right|=\left|\operatorname{Aut}_{\mathcal{L}_{0}}\left(P_{0}\right)\right| \cdot\left|\Gamma^{\prime} / \Gamma_{0}\right|=\left|\operatorname{Aut}_{\mathcal{L}_{0}}\left(P_{0}^{*}\right)\right| \cdot\left|\Gamma^{\prime} / \Gamma_{0}\right| .
$$


Since $P_{0}^{*}$ is fully normalized in $\mathcal{F}_{0}, S^{*} \in \operatorname{Syl}_{p}\left(\Gamma^{\prime}\right)$, and $S_{0} \in \operatorname{Syl}_{p}\left(\Gamma_{0}\right)$, this shows that $\delta\left(N_{S}\left(Q_{0}\right)\right)$ is a Sylow $p$-subgroup of $\operatorname{Aut}_{\mathcal{L}}\left(Q_{0}\right)$.

Fix some $\psi \in \operatorname{Iso}_{\mathcal{L}}\left(P_{0}, Q_{0}\right)$. Then $\psi \delta\left(N_{S}\left(P_{0}\right)\right) \psi^{-1}$ is a $p$-subgroup of $\operatorname{Aut}_{\mathcal{L}}\left(Q_{0}\right)$. Choose $\chi \in \operatorname{Aut}_{\mathcal{L}}\left(Q_{0}\right)$ such that $(\chi \psi) \delta\left(N_{S}\left(P_{0}\right)\right)(\chi \psi)^{-1} \leq \delta\left(N_{S}\left(Q_{0}\right)\right)$. By definition of the category $\mathcal{L}, \chi \psi$ extends to a morphism $\bar{\psi} \in \operatorname{Mor}_{\mathcal{L}}\left(P, N_{S}\left(Q_{0}\right)\right)$. Set $P^{\prime}=$ $\pi(\bar{\psi})(P)$. Then $P_{0}^{\prime}=Q_{0}, P^{\prime}$ is $\mathcal{F}$-conjugate to $P$, and $P^{\prime} \in \mathcal{H}$ since $P_{0}^{\prime} \in \mathcal{H}_{0}\left(\mathcal{H}_{0}\right.$ is closed under $\mathcal{F}_{0}$-conjugacy). This finishes the proof of (6).

Step 4 . We are now ready to show that $\mathcal{F}$ is $\mathcal{H}$-saturated. For each $P \in \mathcal{H}$, set

$$
E(P)=\operatorname{Ker}\left[\operatorname{Aut}_{\mathcal{L}}(P) \stackrel{\pi_{P}}{\longrightarrow} \operatorname{Aut}_{\mathcal{F}}(P)\right] .
$$

Fix $P \in \mathcal{H}$ such that $\delta\left(N_{S}\left(P_{0}\right)\right) \in \operatorname{Syl}_{p}\left(\operatorname{Aut}_{\mathcal{L}}\left(P_{0}\right)\right)$. By ([6), every subgroup in $\mathcal{H}$ is $\mathcal{F}$-conjugate to some such $P$. Write $G=\operatorname{Aut}_{\mathcal{L}}\left(P_{0}\right), T=\delta\left(N_{S}\left(P_{0}\right)\right)$, and $\widehat{P}=$ $\delta(P)$ for short, where $\delta=\delta_{P_{0}}$ is injective by construction. Thus $\widehat{P} \leq T \in \operatorname{Syl}_{p}(G)$. Fix $R \in \operatorname{Syl}_{p}\left(N_{G}(\widehat{P})\right)$, and choose $\alpha \in G$ such that $\alpha R \alpha^{-1} \leq T$. Then

$$
\alpha R \alpha^{-1} \in \operatorname{Syl}_{p}\left(N_{G}\left(\alpha \widehat{P} \alpha^{-1}\right)\right), \quad \alpha R \alpha^{-1} \leq T \quad \Longrightarrow \quad \alpha R \alpha^{-1}=N_{T}\left(\alpha \widehat{P} \alpha^{-1}\right) .
$$

Also, $\alpha \widehat{P} \alpha^{-1} \leq \alpha R \alpha^{-1} \leq T=\delta\left(N_{S}\left(P_{0}\right)\right)$, and we choose $P^{\prime}=\delta^{-1}\left(\alpha \widehat{P} \alpha^{-1}\right)$. Then

$$
\begin{aligned}
& N_{T}\left(\alpha \widehat{P} \alpha^{-1}\right)=N_{\delta\left(N_{S}\left(P_{0}\right)\right)}\left(\delta\left(P^{\prime}\right)\right)=\delta\left(N_{S}\left(P^{\prime}\right)\right), \\
& N_{G}\left(\alpha \widehat{P} \alpha^{-1}\right)=N_{\operatorname{Aut}_{\mathcal{L}}\left(P_{0}\right)}\left(\delta\left(P^{\prime}\right)\right)=\operatorname{Aut}_{\mathcal{L}}\left(P^{\prime}\right) .
\end{aligned}
$$

We have thus found $P^{\prime} \mathcal{F}$-conjugate to $P$ such that $\delta\left(N_{S}\left(P^{\prime}\right)\right) \in \operatorname{Syl}_{p}\left(\operatorname{Aut}_{\mathcal{L}}\left(P^{\prime}\right)\right)$. This in turn implies

$$
\operatorname{Aut}_{S}\left(P^{\prime}\right) \in \operatorname{Syl}_{p}\left(\operatorname{Aut}_{\mathcal{F}}\left(P^{\prime}\right)\right) \quad \text { and } \quad \delta\left(C_{S}\left(P^{\prime}\right)\right) \in \operatorname{Syl}_{p}\left(E\left(P^{\prime}\right)\right) .
$$

Since $\mathcal{F}_{2}$ is a full subcategory of $\mathcal{F}$, all $\mathcal{F}$-morphisms between subgroups in $\mathcal{H}$ lift to morphisms in $\mathcal{L}$ by definition of $\mathcal{F}_{2}$. Since $P^{\prime} \in \mathcal{H},\left|\operatorname{Aut}_{\mathcal{F}}\left(P^{\prime}\right)\right|$ and $\left|E\left(P^{\prime}\right)\right|$ depend only on the $\mathcal{F}$-conjugacy class $(=\mathcal{L}$-isomorphism class $)$ of $P^{\prime}$. So by (7), the subgroup $P^{\prime}$ must be fully normalized and fully centralized in $\mathcal{F}$. If $P^{\prime \prime} \in \mathcal{H}$ is any other subgroup $\mathcal{F}$-conjugate to $P^{\prime}$ and fully normalized, then since $\left|N_{S}\left(P^{\prime \prime}\right)\right|=\left|\operatorname{Aut}_{S}\left(P^{\prime \prime}\right)\right| \cdot\left|C_{S}\left(P^{\prime \prime}\right)\right|, P^{\prime \prime}$ must be fully centralized and $\operatorname{Aut}_{S}\left(P^{\prime \prime}\right) \in$ $\operatorname{Syl}_{p}\left(\operatorname{Aut}_{\mathcal{F}}\left(P^{\prime \prime}\right)\right)$. This finishes the proof of axiom (I) for the fusion system $\mathcal{F}$ and also shows that a subgroup $P \in \mathcal{H}$ is fully centralized if and only if $\delta_{P}\left(C_{S}(P)\right) \in$ $\operatorname{Syl}_{p}(E(P))$.

Now assume $P \in \mathcal{H}$ is fully centralized in $\mathcal{F}$. Thus $\delta\left(C_{S}(P)\right) \in \operatorname{Syl}_{p}(E(P))$. Fix $Q \in \mathcal{H}$ and $\varphi \in \operatorname{Iso}_{\mathcal{F}}(Q, P)$, and set

$$
N=N_{\varphi}=\left\{g \in N_{S}(Q) \mid \varphi c_{g} \varphi^{-1} \in \operatorname{Aut}_{S}(P)\right\} .
$$

Choose some $\psi \in \operatorname{Iso}_{\mathcal{L}}(Q, P)$ in $\pi^{-1}(\varphi)$. Then $\psi \delta_{Q}(N) \psi^{-1} \leq \delta_{P}\left(N_{S}(P)\right) \cdot E(P)$. Since $\delta_{P}\left(C_{S}(P)\right) \in \operatorname{Syl}_{p}(E(P)), \psi \delta_{Q}(N) \psi^{-1}$ is conjugate by an element of $E(P)$ to a subgroup of $\delta_{P}\left(N_{S}(P)\right)$. So upon replacing $\psi$ by $\chi \psi$ for some appropriate $\chi \in E(P)$, we can assume $\psi \delta_{Q}(N) \psi^{-1} \leq \delta_{P}\left(N_{S}(P)\right)$.

Set $\psi_{0}=\psi$, regarded as an element $\psi_{0} \in \operatorname{Iso}_{\mathcal{L}_{1}}\left(Q_{0}, P_{0}\right)$. By definition of $\mathcal{L}$, $\delta_{Q}$, and $\delta_{P}$, upon restricting to intersections with $S_{0}$, the inclusion $\psi \delta_{Q}(N) \psi^{-1} \leq$ $\delta_{P}\left(N_{S}(P)\right)$ implies

$$
\psi_{0} \delta_{Q_{0}}(N) \psi_{0}^{-1} \leq \delta_{P_{0}}\left(N_{S}(P)\right)
$$

in $\operatorname{Aut}_{\mathcal{L}_{1}}\left(P_{0}\right)$. By (4), for each $g \in N$, there is a unique element $\bar{\varphi}(g) \in N_{S}(P)$ such that $\psi_{0} \delta_{Q_{0}}(g) \psi_{0}^{-1}=\delta_{P_{0}}(\bar{\varphi}(g))$. Then $\bar{\varphi} \in \operatorname{Hom}\left(N, N_{S}(P)\right)$. We claim $\bar{\varphi}$ is a 
morphism in $\mathcal{F}$. By (5), there is $\bar{\psi}_{0} \in \operatorname{Mor}_{\mathcal{L}_{1}}\left(N_{0}, N_{S_{0}}(P)\right)$ such that $\left.\bar{\psi}_{0}\right|_{Q_{0}, P_{0}}=\psi_{0}$. By the uniqueness of extensions (4), for all $g \in N$,

$$
\bar{\psi}_{0} \circ \delta_{N_{0}}(g)=\delta_{N_{S_{0}}(P)}(\bar{\varphi}(g)) \circ \bar{\psi}_{0}
$$

in $\mathcal{L}_{1}$, and hence $\bar{\varphi} \in \operatorname{Hom}_{\mathcal{F}}\left(N, N_{S}(P)\right)$ by the definition of $\mathcal{F}$ in Step 2 .

Step 5. By Step $4, \mathcal{F}$ is $\mathcal{H}$-saturated; i.e., it satisfies the saturation axioms for subgroups in $\mathcal{H}$. It is also $\mathcal{H}$-generated by definition: each morphism in $\mathcal{F}$ is a composite of restrictions of morphisms between subgroups in $\mathcal{H}$. So by [4, Theorem A], to prove $\mathcal{F}$ is saturated, it suffices to show the following holds for all $P \leq S$ :

$$
\begin{aligned}
P \mathcal{F} \text {-centric, } P & \notin \mathcal{H} \Longrightarrow \\
& \exists P^{\prime} \mathcal{F} \text {-conj. to } P, \operatorname{Aut}_{S}\left(P^{\prime}\right) \cap O_{p}\left(\operatorname{Aut}_{\mathcal{F}}\left(P^{\prime}\right)\right) \supsetneqq \operatorname{Inn}\left(P^{\prime}\right) .
\end{aligned}
$$

Let $\mathcal{K}$ be the set of all $P \leq S$ such that the saturation axioms hold for subgroups $\mathcal{F}$-conjugate to $P$ and all of their overgroups. Since $\mathcal{H}_{0}$ is closed under overgroups and $\mathcal{F}_{0}$-conjugacy by assumption, $\mathcal{H}$ is closed under overgroups and $\mathcal{F}$-conjugacy by construction, and thus $\mathcal{K} \supseteq \mathcal{H}$. Let $\mathcal{K}^{*}$ be the set of subgroups of $S$ not in $\mathcal{K}$, and let $\mathcal{K}_{0}^{*}$ be the set of subgroups of $S_{0}$ not in $\mathcal{K}$. We must show $\mathcal{K}^{*}=\varnothing$. This will be done by first proving that for all $P \leq S$,

$$
P_{0} \in \mathcal{K} \text { or } P_{0} \text { maximal in } \mathcal{K}_{0}^{*} \Longrightarrow(8) \text { holds for } P \text {. }
$$

Fix $P$ as in (91). We first show that there exists $P^{*} \mathcal{F}$-conjugate to $P$ such that $P_{0}^{*}$ is fully normalized in $\mathcal{F}_{0}$. If $P_{0}$ is fully normalized, we are done, so assume otherwise. Let $P_{0}^{\prime}$ be $\mathcal{F}_{0}$-conjugate to $P_{0}$ and fully normalized in $\mathcal{F}_{0}$. By [3, Proposition A.2(b)], there is $\rho \in \operatorname{Hom}_{\mathcal{F}_{0}}\left(N_{S_{0}}\left(P_{0}\right), N_{S_{0}}\left(P_{0}^{\prime}\right)\right)$ such that $\rho\left(P_{0}\right)=P_{0}^{\prime}$. Clearly, $P_{0} \supsetneqq S_{0}$, so $N_{S_{0}}\left(P_{0}\right) \supsetneqq P_{0}$. Whether $P_{0} \in \mathcal{K}$ or $P_{0}$ is maximal in $\mathcal{K}_{0}^{*}, N_{S_{0}}\left(P_{0}\right) \in \mathcal{K}$, and hence the saturation axioms hold for $N_{S_{0}}\left(P_{0}\right), N_{S_{0}}\left(P_{0}^{\prime}\right)$, and all subgroups of $S$ which contain them.

Set $R=N_{S_{0}}\left(P_{0}\right)$ and $R^{\prime}=\rho(R)$. Choose $R^{\prime \prime} \leq S_{0}$ and $\tau \in \operatorname{Iso}_{\mathcal{F}}\left(R, R^{\prime \prime}\right)$ such that $R^{\prime \prime}$ is fully normalized in $\mathcal{F}$, and set $P_{0}^{\prime \prime}=\tau\left(P_{0}\right)$. In general, for a pair of subgroups $Q_{1} \leq Q \leq S$, we write $\operatorname{Aut}_{\mathcal{F}}\left(Q: Q_{1}\right)$ for the group of elements in $\operatorname{Aut}_{\mathcal{F}}(Q)$ which leave $Q_{1}$ invariant, and similarly for $\operatorname{Aut}_{S}\left(Q: Q_{1}\right)$ and $N_{S}\left(Q: Q_{1}\right)=N_{S}(Q) \cap N_{S}\left(Q_{1}\right)$. Since $\operatorname{Aut}_{S}\left(R^{\prime \prime}\right) \in \operatorname{Syl}_{p}\left(\operatorname{Aut}_{\mathcal{F}}\left(R^{\prime \prime}\right)\right)$ (recall $R^{\prime \prime}$ is fully normalized in $\left.\mathcal{F}\right)$, there is $\omega \in \operatorname{Aut}_{\mathcal{F}}\left(R^{\prime \prime}\right)$ such that $\operatorname{Aut}_{S}\left(R^{\prime \prime}: \omega\left(P_{0}^{\prime \prime}\right)\right) \in \operatorname{Syl}_{p}\left(\operatorname{Aut}_{\mathcal{F}}\left(R^{\prime \prime}: \omega\left(P_{0}^{\prime \prime}\right)\right)\right)$. So upon replacing $\tau$ by $\omega \tau$, we can assume $\operatorname{Aut}_{S}\left(R^{\prime \prime}: P_{0}^{\prime \prime}\right) \in \operatorname{Syl}_{p}\left(\operatorname{Aut}_{\mathcal{F}}\left(R^{\prime \prime}: P_{0}^{\prime \prime}\right)\right)$.

In particular, there is $\chi \in \operatorname{Aut}_{\mathcal{F}}\left(R^{\prime \prime}: P_{0}^{\prime \prime}\right)$ such that $(\chi \tau) \operatorname{Aut}_{S}\left(R: P_{0}\right)(\chi \tau)^{-1} \leq$ $\operatorname{Aut}_{S}\left(R^{\prime \prime}: P_{0}^{\prime \prime}\right)$. Since $\mathcal{F}$ is $\mathcal{H}$-saturated and $R^{\prime \prime} \in \mathcal{H}$ (and is fully centralized), $\chi \tau$ extends to some $\bar{\tau} \in \operatorname{Hom}_{\mathcal{F}}\left(N_{S}\left(R: P_{0}\right), N_{S}\left(R^{\prime \prime}: P_{0}^{\prime \prime}\right)\right)$. Note that $P \leq N_{S}\left(R: P_{0}\right)$ and $\bar{\tau}\left(P_{0}\right)=\tau\left(P_{0}\right)=P_{0}^{\prime \prime}$. Set $P^{\prime \prime}=\bar{\tau}(P)$. By a similar argument, there is $\chi^{\prime} \in \operatorname{Aut}_{\mathcal{F}}\left(R^{\prime \prime}: P_{0}^{\prime \prime}\right)$ such that $\chi^{\prime} \tau \rho^{-1} \in \operatorname{Iso}_{\mathcal{F}}\left(R^{\prime}, R^{\prime \prime}\right)$ extends to some morphism $\bar{\rho} \in \operatorname{Hom}_{\mathcal{F}}\left(N_{S}\left(R^{\prime}: P_{0}^{\prime}\right), N_{S}\left(R^{\prime \prime}: P_{0}^{\prime \prime}\right)\right)$, where $\bar{\rho}\left(P_{0}^{\prime}\right)=\tau \rho^{-1}\left(P_{0}^{\prime}\right)=P_{0}^{\prime \prime}$.

We claim that

$$
\left|N_{S_{0}}\left(P_{0}\right)\right|<\left|N_{S_{0}}\left(R^{\prime}: P_{0}^{\prime}\right)\right| \leq\left|N_{S_{0}}\left(R^{\prime \prime}: P_{0}^{\prime \prime}\right)\right| \leq\left|N_{S_{0}}\left(P_{0}^{\prime \prime}\right)\right| .
$$

Since $P_{0}$ is not fully normalized in $\mathcal{F}_{0}, R^{\prime}=\rho\left(N_{S_{0}}\left(P_{0}\right)\right) \supsetneqq N_{S_{0}}\left(P_{0}^{\prime}\right)$, and hence

$$
R^{\prime} \supsetneqq N_{N_{S_{0}}\left(P_{0}^{\prime}\right)}\left(R^{\prime}\right)=N_{S_{0}}\left(R^{\prime}: P_{0}^{\prime}\right) .
$$

This proves the first inequality in (10). The next one holds since $\bar{\rho}$ sends $N_{S_{0}}\left(R^{\prime}: P_{0}^{\prime}\right)$ into $N_{S_{0}}\left(R^{\prime \prime}: P_{0}^{\prime \prime}\right)$, and the last since all elements of $N_{S_{0}}\left(R^{\prime \prime}: P_{0}^{\prime \prime}\right)$ normalize $P_{0}^{\prime \prime}$. Thus $P^{\prime \prime}$ is $\mathcal{F}$-conjugate to $P$ and $\left|N_{S_{0}}\left(P_{0}^{\prime \prime}\right)\right|>\left|N_{S_{0}}\left(P_{0}\right)\right|$. If $P_{0}^{\prime \prime}$ is not fully normalized 
in $\mathcal{F}_{0}$, then we repeat this procedure, until we do find a subgroup $P^{*}$ which is $\mathcal{F}$-conjugate to $P$ and such that $P_{0}^{*}$ is fully normalized in $\mathcal{F}_{0}$.

We are now ready to prove (9). Assume $P$ is $\mathcal{F}$-centric and $P \notin \mathcal{H}$; otherwise the statement is empty. Thus $P_{0} \notin \mathcal{H}_{0}=\mathrm{Ob}\left(\mathcal{L}_{0}\right)$. By definition of a linking system, either $P_{0}$ and $P_{0}^{*}$ are not $\mathcal{F}_{0}$-centric or they are not $\mathcal{F}_{0}$-radical. If $P_{0}^{*}$ is not $\mathcal{F}_{0^{-}}$ centric, then there is $g \in C_{S_{0}}\left(P_{0}^{*}\right) \backslash P_{0}^{*}$ (since $P_{0}^{*}$ is fully centralized). If $P_{0}^{*}$ is not $\mathcal{F}_{0}$-radical, then $O_{p}\left(\operatorname{Aut}_{\mathcal{F}_{0}}\left(P_{0}^{*}\right)\right) \supsetneqq \operatorname{Inn}\left(P_{0}^{*}\right)$ and is contained in the Sylow subgroup $\operatorname{Aut}_{S_{0}}\left(P_{0}^{*}\right)$ ( $P_{0}^{*}$ is fully normalized), and thus there is $g \in N_{S_{0}}\left(P_{0}^{*}\right) \backslash P_{0}^{*}$ such that $c_{g} \in O_{p}\left(\operatorname{Aut}_{\mathcal{F}_{0}}\left(P_{0}^{*}\right)\right)$. In either case,

$$
g \in Q^{*} \stackrel{\text { def }}{=}\left\{g \in N_{S_{0}}\left(P_{0}^{*}\right) \mid c_{g} \in O_{p}\left(\operatorname{Aut}_{\mathcal{F}_{0}}\left(P_{0}^{*}\right)\right)\right\} \quad \text { and } \quad g \notin P_{0}^{*},
$$

and hence $Q^{*} \supsetneqq P_{0}^{*}$. Also, $P^{*}$ normalizes $Q^{*}$ and $P^{*} Q^{*} \supsetneqq P^{*}$, so $N_{P^{*} Q^{*}}\left(P^{*}\right) \supsetneqq P^{*}$, and there is $x \in Q^{*} \backslash P^{*}$ such that $x \in N_{S_{0}}\left(P^{*}\right)$. For any such $x, c_{x} \notin \operatorname{Inn}\left(P^{*}\right)$ $\left(C_{S}\left(P^{*}\right) \leq P^{*}\right.$ since $P$ is $\mathcal{F}$-centric), but $\left.c_{x}\right|_{P_{0}^{*}}$ is in $O_{p}\left(\operatorname{Aut}_{\mathcal{F}_{0}}\left(P_{0}^{*}\right)\right)$ and $c_{x}$ induces the identity on $P^{*} / P_{0}^{*}\left(\right.$ since $\left.x \in S_{0}\right)$. Hence $c_{x} \in O_{p}\left(\operatorname{Aut}_{\mathcal{F}}\left(P^{*}\right)\right)$ : the subgroup

$$
\left\{\alpha \in \operatorname{Aut}_{\mathcal{F}}\left(P^{*}\right)|\alpha|_{P_{0}^{*}} \in O_{p}\left(\operatorname{Aut}_{\mathcal{F}_{0}}\left(P_{0}^{*}\right)\right), \alpha \text { induces the identity on } P^{*} / P_{0}^{*}\right\}
$$

is a normal $p$-subgroup of $\operatorname{Aut}_{\mathcal{F}}\left(P^{*}\right)$ since the group of all $\alpha \in \operatorname{Aut}\left(P^{*}\right)$ which induce the identity on $P_{0}^{*}$ and on $P^{*} / P_{0}^{*}$ is a $p$-group (cf. [7, Corollary 5.3.3]). Thus (8) holds for $P$, and this finishes the proof of (9).

We want to show that $\mathcal{F}$ is saturated, i.e., that $\mathcal{K}^{*}=\varnothing$. Assume otherwise; then $\mathcal{K}_{0}^{*} \neq \varnothing$ since $P \in \mathcal{K}^{*}$ implies $P_{0} \in \mathcal{K}_{0}^{*}$. Choose $Q$ to be maximal in $\mathcal{K}_{0}^{*}$, and choose $P$ to be maximal among those $P \in \mathcal{K}^{*}$ such that $P_{0}=Q$. Then $P$ is also maximal in $\mathcal{K}^{*}$. By [4, Lemmas $2.4 \& 2.5$ ], this maximality of $P$ among subgroups not satisfying the saturation axioms implies (8) does not hold for $P$. Since this contradicts (9), we now conclude that $\mathcal{K}^{*}=\varnothing$, and hence that $\mathcal{F}$ is saturated and (81) holds for all $P$.

Now that we know $\mathcal{F}$ is saturated, (8) implies that $\mathcal{H}$ contains all subgroups which are $\mathcal{F}$-centric and $\mathcal{F}$-radical. Also, $\mathcal{F}_{0}$ is normal in $\mathcal{F}$ (Definition 7 ).

Step 6. We show here that (31) implies (3), and that (2) and (3) imply $E(P)$ is a $p$-group for all $P \in \mathcal{H}$. Assume first (31) holds. Fix $P \in \mathcal{H}_{0}, P \leq Q \leq P \cdot C_{S_{0}}(P)$, and $\alpha \in \operatorname{Aut}_{\mathcal{F}_{1}}(Q)$ such that $\left.\alpha\right|_{P}=$ Id. If $P$ is $\mathcal{F}_{0}$-centric, then $Q=P$ and $\alpha=$ Id. If $\Gamma / \Gamma_{0}$ is a $p$-group, then $\operatorname{Aut}_{\mathcal{L}_{0}}(Q)$ has $p$-power index in $\operatorname{Aut}_{\mathcal{L}_{1}}(Q)$ (there is a homomorphism from $\operatorname{Aut}_{\mathcal{L}_{1}}(Q)$ to $\Gamma / \Gamma_{0}$ sending $\llbracket \varphi, \gamma \rrbracket$ to $\gamma \Gamma_{0}$ with kernel $\left.\operatorname{Aut}_{\mathcal{L}_{0}}(Q)\right)$; so $\operatorname{Aut}_{\mathcal{F}_{0}}(Q)$ has $p$-power index in $\operatorname{Aut}_{\mathcal{F}_{1}}(Q)$, and hence $\alpha^{p^{k}} \in$ $\operatorname{Aut}_{\mathcal{F}_{0}}(Q)$ for some $k$. By Proposition $4(\mathrm{~g}), P$ is $\mathcal{F}_{0}$-quasicentric, and so $\alpha^{p^{k}}$ has $p$-power order. Thus $\alpha$ has $p$-power order in both cases, and this proves (3).

For the rest of the proof, we assume (22) and (3) hold. We next show $E(P)$ is a $p$-group when $P \in \mathcal{H}_{0}$, i.e., when $P \leq S_{0}$. Assume otherwise, and let $P$ be maximal among those $P \in \mathcal{H}_{0}$ for which $E(P)$ is not a $p$-group. Since this depends only on the $\mathcal{F}_{0}$-conjugacy class of $P$, we can assume $P$ is fully normalized in $\mathcal{F}_{0}$. Since $E\left(S_{0}\right)=C_{\Gamma}\left(S_{0}\right)$ is a $p$-group by (2), we have $P \supsetneqq S_{0}$.

Fix Id $\neq \psi \in E(P)$ of order prime to $p$. Write $\psi=\llbracket \varphi, \gamma \rrbracket$, where $\gamma \in \Gamma$ and $\varphi \in \operatorname{Iso}_{\mathcal{L}_{0}}(\gamma(P), P)$. Thus $\pi\left(\varphi^{-1}\right)=\left.\pi(\gamma)\right|_{P, \gamma(P)} \in \operatorname{Hom}_{\mathcal{F}_{0}}(P, \gamma(P))$. Set $N=N_{S_{0}}(P) \supsetneqq P$. For each $g \in N$,

$$
\pi\left(\varphi^{-1}\right) \circ c_{g}=\left.\left(\pi(\gamma) \circ c_{g}\right)\right|_{P, \gamma(P)}=\left.\left(c_{\gamma(g)} \circ \pi(\gamma)\right)\right|_{P, \gamma(P)}=c_{\gamma(g)} \circ \pi\left(\varphi^{-1}\right) .
$$


By axiom (A), and since $P$ is fully normalized in $\mathcal{F}_{0}$, there is $x \in C_{S_{0}}(P) \leq N$ such that $\varphi^{-1} \circ \delta_{0}(g x)=\delta_{0}(\gamma(g)) \circ \varphi^{-1}$ in $\mathcal{L}_{0}$. Thus $\varphi \delta_{0}(\gamma(N)) \varphi^{-1}=\delta_{0}(N)$.

By Proposition 4 (e) (applied to $\mathcal{L}_{0}$ ), $\varphi$ extends to some $\bar{\varphi} \in \operatorname{Iso}_{\mathcal{L}_{0}}(\gamma(N), N)$, and hence $\psi$ also extends to an automorphism $\bar{\psi} \in \operatorname{Aut}_{\mathcal{L}}(N)$. By the uniqueness of the extension, $\bar{\psi}$ has the same order as $\psi$, which is thus prime to $p$. Set $\alpha=\pi(\bar{\psi}) \in$ $\operatorname{Aut}_{\mathcal{F}}(N)$. By (3), $\left.\alpha\right|_{P \cdot C_{N}(P)}$ has $p$-power order, hence is the identity since $\alpha$ has order prime to $p$. For all $g \in N$ and $x \in P, \alpha(g) x \alpha(g)^{-1}=\alpha\left(g x g^{-1}\right)=g x g^{-1}$ since $\left.\alpha\right|_{P}=\mathrm{Id}$, and hence $g^{-1} \alpha(g) \in C_{N}(P)$. Thus $\alpha$ induces the identity on $N / C_{N}(P)$ and on $C_{N}(P)$, so $\alpha$ has $p$-power order by [7, Corollary 5.3.3], and hence $\alpha=$ Id. This proves that $\bar{\psi} \in E(N)$, which contradicts the assumption that $P$ was maximal among subgroups in $\mathcal{H}_{0}$ with $E(P)$ not a $p$-group.

Thus $E(P)$ is a $p$-group for all $P \in \mathcal{H}_{0}$. Now assume $P \in \mathcal{H} \backslash \mathcal{H}_{0}$. Fix $\operatorname{Id} \neq \psi \in$ $\operatorname{Aut}_{\mathcal{L}}(P)$ of order prime to $p$ such that $\pi_{P}(\psi)=\operatorname{Id}_{P}$, and set $\psi_{0}=\left.\psi\right|_{P_{0}, P_{0}}$. Then $\psi_{0} \in E\left(P_{0}\right), E\left(P_{0}\right)$ is a $p$-group, and hence $\psi_{0}=\operatorname{Id}$. But then $\psi, \operatorname{Id} \in \operatorname{Aut}_{\mathcal{L}}(P)$ are two automorphisms with the same restriction to $\operatorname{Aut}_{\mathcal{L}}\left(P_{0}\right)$, which contradicts the definition of $\operatorname{Aut}_{\mathcal{L}}(P)$ in Step 2.

Step 7. We now prove that $\mathcal{L}$ is a linking system associated to $\mathcal{F}$ by checking the axioms in Definition 3. We first claim that for each $P, Q \in \mathcal{H}$ such that $P$ is fully centralized in $\mathcal{F}$,

$$
\delta_{P}\left(C_{S}(P)\right) \text { acts freely on } \operatorname{Mor}_{\mathcal{L}}(P, Q) \text { and } \pi_{P, Q} \text { is the orbit map. }
$$

Since every morphism in $\mathcal{L}$ (and also in $\mathcal{F}$ ) factors uniquely as the composite of an isomorphism followed by an inclusion, it suffices to prove this when $P$ and $Q$ are $\mathcal{F}$-conjugate. It thus suffices to prove it when $P=Q$, and this follows from (7) $\left(\delta_{P}\left(C_{S}(P)\right) \in \operatorname{Syl}_{p}(E(P))\right)$ and Step $6(E(P)$ is a $p$-group $)$.

This proves the last part of axiom (A). The rest of axiom (A) holds by construction (note that two objects of $\mathcal{L}$ are $\mathcal{L}$-isomorphic whenever they are $\mathcal{F}$-isomorphic), and by Step 5 (all objects in $\mathcal{L}$ are $\mathcal{F}$-centric and $\mathcal{F}$-radical). Also, axiom (B) holds by construction, and $(\mathrm{C})$ holds by definition of the functor $\pi: \mathcal{L} \longrightarrow \mathcal{F}$.

The pair $\mathcal{L}_{0} \subseteq \mathcal{L}$ clearly satisfies the conditions in Definition 8, and so $\mathcal{L}_{0}$ is normal in $\mathcal{L}$.

Step 8. Now assume $\mathcal{L}^{\prime}$ is another linking system with the same objects, associated to a fusion system $\mathcal{F}^{\prime}$ over $S$, with $\mathcal{L}_{0} \unlhd \mathcal{L}^{\prime}$, and where $\operatorname{Aut}_{\mathcal{L}^{\prime}}\left(S_{0}\right)=\Gamma$ with the same conjugation action on $\mathcal{L}_{0}$. Let $\mathcal{F}_{1}^{\prime} \subseteq \mathcal{F}^{\prime}$ and $\mathcal{L}_{1}^{\prime} \subseteq \mathcal{L}^{\prime}$ be the full subcategories with $\operatorname{Ob}\left(\mathcal{F}_{1}^{\prime}\right)=\left\{P \leq S_{0}\right\}$ and $\operatorname{Ob}\left(\mathcal{L}_{1}^{\prime}\right)=\mathcal{H}_{0}$. Then $\mathcal{F}_{1}^{\prime}$ and $\mathcal{F}_{1}$ are both fusion systems over $S_{0}$ (not necessarily saturated). By condition (ii) in the definition of a normal fusion system, $\mathcal{F}_{1}^{\prime}$ and $\mathcal{F}_{1}$ are both generated (as fusion systems) by $\mathcal{F}_{0}$ and $\operatorname{Aut}_{\Gamma}\left(S_{0}\right)$, and hence $\mathcal{F}_{1}^{\prime}=\mathcal{F}_{1}$.

Define $\Phi_{1}: \mathcal{L}_{1} \longrightarrow \mathcal{L}_{1}^{\prime}$ to be the functor which is the identity on objects, and which sends $\llbracket \varphi, \gamma \rrbracket \in \operatorname{Mor}_{\mathcal{L}_{1}}(P, Q)$ to $\left.\varphi \circ \gamma\right|_{P, \gamma(P)} \in \operatorname{Mor}_{\mathcal{L}_{1}^{\prime}}(P, Q)$. This preserves composition (hence is a functor) by the assumption that $\mathcal{L}$ and $\mathcal{L}^{\prime}$ induce the same $\Gamma$-actions on $\mathcal{L}_{0}$. If $P$ is fully centralized in $\mathcal{F}$, then it is fully centralized in $\mathcal{F}^{\prime}$ since it has the same $\mathcal{F}$ - and $\mathcal{F}^{\prime}$-conjugacy classes. By axiom (A) (applied to $\mathcal{L}$ and $\left.\mathcal{L}^{\prime}\right), C_{S}(P)$ acts freely on $\operatorname{Mor}_{\mathcal{L}}(P, Q)$ and on $\operatorname{Mor}_{\mathcal{L}^{\prime}}(P, Q)$ with orbit sets $\operatorname{Hom}_{\mathcal{F}}(P, Q)=\operatorname{Hom}_{\mathcal{F}^{\prime}}(P, Q)$. By construction, $\left(\Phi_{1}\right)_{P, Q}$ is equivariant with respect to these actions, and hence is also a bijection. If $P$ is not fully centralized, then choose $\psi \in \operatorname{Iso}_{\mathcal{L}}\left(P^{*}, P\right)$ such that $P^{*}$ is fully centralized; $\Phi_{1}(\psi)$ is an isomorphism 
in $\mathcal{L}^{\prime}$, composition with $\psi$ and $\Phi_{1}(\psi)$ sends $\operatorname{Mor}_{\mathcal{L}}(P, Q)$ bijectively to $\operatorname{Mor}_{\mathcal{L}}\left(P^{*}, Q\right)$ and similarly for $\mathcal{L}^{\prime}$, and thus $\left(\Phi_{1}\right)_{P, Q}$ is again bijective. So $\Phi_{1}$ is an isomorphism of categories $\mathcal{L}_{1} \cong \mathcal{L}_{1}^{\prime}$.

For each $P, Q \in \mathcal{H}$, consider the restriction homomorphism

$$
\operatorname{Mor}_{\mathcal{L}^{\prime}}(P, Q) \stackrel{\operatorname{Res}_{P, Q}}{\longrightarrow} \operatorname{Mor}_{\mathcal{L}^{\prime}}\left(P_{0}, Q_{0}\right)
$$

This is injective by Proposition 4 (e) (the uniqueness part), and

$$
\begin{aligned}
& \operatorname{Im}\left(\operatorname{Res}_{P, Q}\right)= \\
& \quad\left\{\psi \in \operatorname{Mor}_{\mathcal{L}^{\prime}}\left(P_{0}, Q_{0}\right) \mid \forall g \in P \exists h \in Q \text { with } \psi \circ \delta_{P_{0}}(g)=\delta_{Q_{0}}(h) \circ \psi\right\}
\end{aligned}
$$

by Proposition $4(\mathrm{e})$ and axiom (C). Using this and the definition of $\mathcal{L}$ in Step 2, $\Phi_{1}$ extends to an isomorphism $\Phi: \mathcal{L} \longrightarrow \mathcal{L}^{\prime}$ of linking systems. By axiom $(\mathrm{C})$, $\operatorname{Hom}_{\mathcal{F}}(P, Q)$ is determined by (12) for each $P, Q$, and thus $\mathcal{F}^{\prime}=\mathcal{F}$.

In general, the uniqueness of the extension of fusion systems in Theorem 9 does not follow from the information about the fusion systems alone. For example, let $\mathcal{F}_{0}$ be the fusion system of $A_{6}$ (over $S_{0} \cong D_{8}$ ). Set $S=C_{2} \times S_{0}$, identified as a Sylow 2-subgroup of $C_{2} \times A_{6}$ and also of $\Sigma_{6}$. Then $\mathcal{F}=\mathcal{F}_{S}\left(C_{2} \times A_{6}\right)$ and $\mathcal{F}^{\prime}=$ $\mathcal{F}_{S}\left(\Sigma_{6}\right)$ are both fusion systems over $S$ containing $\mathcal{F}_{0}$ as a normal subsystem, and $\operatorname{Aut}_{\mathcal{F}}\left(S_{0}\right)=\operatorname{Aut}_{\mathcal{F}^{\prime}}\left(S_{0}\right)\left(=\operatorname{Inn}\left(S_{0}\right)\right)$. But these fusion systems are not isomorphic.

Condition (2) in Theorem 9 is clearly necessary to get a linking system, since $C_{\Gamma}\left(S_{0}\right)=\operatorname{Ker}\left(\pi_{S_{0}, S_{0}}\right)$ must be isomorphic to $C_{S}\left(S_{0}\right)$. Condition (3) is necessary since by Proposition $4(\mathrm{~g})$, each $P \in \mathcal{H}=\mathrm{Ob}(\mathcal{L})$ must be $\mathcal{F}$-quasicentric. If Condition (3) in Theorem 9 fails to hold, and all of the other hypotheses do hold, then one can arrange for (31) (hence (3)) to hold by restricting the objects in $\mathcal{L}_{0}$ to those which are $\mathcal{F}_{0}$-centric. In other words, one can always construct some linking system associated to $\mathcal{F}$ in this situation, but sometimes only after restricting the set of objects.

As remarked earlier, whenever $\mathcal{L}_{0} \unlhd \mathcal{L}$ and $\Gamma_{0} \unlhd \Gamma$ are as in Theorem 9], the geometric realisation $\left|\mathcal{L}_{0}\right|$ has the homotopy type of a covering space of $|\mathcal{L}|$ with covering group $\Gamma / \Gamma_{0}$.

The author would like to give profound thanks to Natàlia Castellana, Assaf Libman, Kasper Andersen, and especially the referee, for having read this paper carefully and having sent many suggestions which helped to greatly improve it.

\section{REFERENCES}

[1] K. Andersen, B. Oliver, \& J. Ventura, Reduced, tame, and exotic fusion systems (preprint)

[2] M. Aschbacher, Normal subsystems of fusion systems, Proc. London Math. Soc. 97 (2008), 239-271 MR2434097

[3] C. Broto, R. Levi, \& B. Oliver, The homotopy theory of fusion systems, J. Amer. Math. Soc. 16 (2003), 779-856 MR.1992826 (2004k:55016)

[4] C. Broto, N. Castellana, J. Grodal, R. Levi, \& B. Oliver, Subgroup families controlling $p$-local finite groups, Proc. London Math. Soc. 91 (2005), 325-354 MR.2167090 (2007e:20111)

[5] C. Broto, N. Castellana, J. Grodal, R. Levi, \& B. Oliver, Extensions of $p$-local finite groups, Trans. Amer. Math. Soc. 359 (2007), 3791-3858 MR2302515(2008i:55013)

[6] N. Castellana \& A. Libman, Wreath products and representations of $p$-local finite groups, Advances in Mathematics 221 (2009), 1302-1344. MR2518640 (2010b:20029)

[7] D. Gorenstein, Finite groups, Harper \& Row (1968) MR0231903 (38:229)

[8] B. Oliver \& J. Ventura, Extensions of linking systems with $p$-group kernel, Math. Annalen 338 (2007), 983-1043 MR2317758 (2008k:55029) 
[9] B. Oliver \& J. Ventura, Saturated fusion systems over 2-groups, Trans. Amer. Math. Soc. 361 (2009), 6661-6728. MR2538610

[10] L. Puig, Frobenius categories, J. Algebra 303 (2006), 309-357 MR2253665 (2007j:20011)

Laboratoire Analyse, Géométrie and Applications, Institut Galilée, Av. J-B Clément, 93430 Villetaneuse, France

E-mail address: bobol@math.univ-paris13.fr 